\title{
Hypoxia-regulated expression of CD44 and osteopontin can change the phenotype of glioma stem-like cells from highly invasive to less invasive/proliferative tumors in glioblastoma
}

\section{Masahiro Nishikawa}

Ehime University Graduate School of Medicine School of Medicine: Ehime Daigaku Daigakuin Igakukei Kenkyuka Igakubu https://orcid.org/0000-0002-1302-7646

Akihiro Inoue ( $\square$ iakihiro3@gmail.com )

Ehime University Graduate School of Medicine https://orcid.org/0000-0002-7438-2369

\section{Takanori Ohnishi}

Washokai Sadamoto Hospital

\section{Hajime Yano}

Ehime University Graduate School of Medicine School of Medicine: Ehime Daigaku Daigakuin Igakukei Kenkyuka Igakubu

\section{Saya Ozaki}

Ehime University Graduate School of Medicine School of Medicine: Ehime Daigaku Daigakuin Igakukei Kenkyuka Igakubu

\section{Yonehiro Kanemura}

National Hospital Organization Osaka National Hospital: Kokuritsu Byoin Kiko Osaka Iryo Center

\section{Shirabe Matsumoto}

Ehime University Graduate School of Medicine School of Medicine: Ehime Daigaku Daigakuin Igakukei Kenkyuka Igakubu

\section{Satoshi Suehiro}

Ehime University Graduate School of Medicine School of Medicine: Ehime Daigaku Daigakuin Igakukei Kenkyuka Igakubu

\section{Yoshihiro Ohtsuka}

Ehime University Graduate School of Medicine School of Medicine: Ehime Daigaku Daigakuin Igakukei Kenkyuka Igakubu

\section{Shohei Kohno}

Ehime University Graduate School of Medicine School of Medicine: Ehime Daigaku Daigakuin Igakukei Kenkyuka Igakubu

\section{Shiro Ohue}

Ehime Prefectural Central Hospital: Ehime Kenritsu Chuo Byoin

\section{Seiji Shigekawa}


Ehime University Graduate School of Medicine School of Medicine: Ehime Daigaku Daigakuin Igakukei Kenkyuka Igakubu

\section{Hideaki Watanabe}

Ehime University Graduate School of Medicine School of Medicine: Ehime Daigaku Daigakuin Igakukei Kenkyuka Igakubu

\section{Riko Kitazawa}

Ehime University Hospital: Ehime Daigaku Igakubu Fuzoku Byoin

\section{Junya Tanaka}

Ehime University Graduate School of Medicine School of Medicine: Ehime Daigaku Daigakuin Igakukei Kenkyuka Igakubu

\section{Takeharu Kunieda}

Ehime University Graduate School of Medicine School of Medicine: Ehime Daigaku Daigakuin Igakukei Kenkyuka Igakubu

\section{Research article}

Keywords: glioma stem cell, glioblastoma, CD44, osteopontin, hypoxia, invasion

Posted Date: September 22nd, 2020

DOI: https://doi.org/10.21203/rs.3.rs-72809/v1

License: (c) (i) This work is licensed under a Creative Commons Attribution 4.0 International License. Read Full License 


\section{Abstract \\ Background}

The poor prognosis of glioblastoma multiforme (GBM) is primarily due to highly invasive and highly migratory glioma stem-like cells (GSCs) in tumors. Upon GBM recurrence or progression, the highly invasive phenotype of GSCs changes to a less-motile, proliferative phenotype, thus generating tumor mass. Elucidating the molecular mechanism underlying this phenotypic transition could lead to the identification of effective molecular targets for treating GBM.

\section{Methods}

We examined mRNA expression of hypoxia-inducible factor (HIF)-1a, HIF-2a, CD44, and osteopontin in GBM tissues and investigated the effect of hypoxia $\left(1 \% \mathrm{O}_{2}\right.$ : severe or $5 \% \mathrm{O}_{2}$ : moderate) on expression of these molecules using two lines of cultured GSCs. We also analyzed the effect of osteopontin on the invasiveness, migration, and proliferation of GSCs under hypoxic conditions. In addition, the effect of CD44 knockdown on tumor growth and survival were investigated in vitro and in vivo using a mouse xenograft model.

\section{Results}

Severe hypoxia upregulates CD44 expression via activation of HIF-1a, inducing GSCs to assume a highly invasive phenotype. In contrast, moderate hypoxia upregulates osteopontin expression via activation of HIF-2a. Osteopontin in turn binds to CD44, simultaneously inhibiting CD44-promoted GSC migration and invasion and stimulating GSC proliferation, resulting in GSCs assuming a less-invasive, highly proliferative phenotype. CD44 knockdown significantly inhibited GSC migration and invasion both in vitro and in vivo. However, although CD44 knockdown did not affect tumor growth in vitro, mouse brain tumors generated from GSCs with CD44 knockdown exhibited diminished invasiveness, and the mice survived significantly longer than control mice. In contrast, siRNA-mediated silencing of the osteopontin gene led to decreased GSC proliferation, but the osteopontin-mediated inhibition of high GSC migratory behavior and invasiveness was diminished.

\section{Conclusion}

The highly invasive phenotype of GSCs can be reversed by switching from severe to moderate hypoxia, leading to less-invasive and proliferative tumors. CD44 and osteopontin, which are expressed in a mutually exclusive manner under severe or moderate hypoxia, play a central role in regulating GSC invasion and proliferation by inducing a phenotypic transition, suggesting that these molecules could be effective targets for treating both primary and recurrent GBM. 


\section{Background}

Glioblastoma multiforme (GBM) is the most common and incurable primary malignant brain tumor, with a median survival of 15 months [1]. Even after maximum resection of the tumors followed by administration of current chemo-radiotherapy, most tumors recur from the periphery of the tumor cavity several months later $[2,3]$. Such early tumor progression might be largely due to glioma stem-like cells (GSCs) that survive in the invasion zone of the tumor periphery after initial multimodal treatment; however, few studies have investigated the pathologic role of GSCs that survive in this area. Munthe et al. reported that glioma cells in the tumor periphery exhibit a stem cell phenotype [4]. Angelucci et al. recently described significant differences between cancer stem cells from the tumor mass and those from peritumoral GBM tissue in terms of proliferation, ultrastructural features, and stemness profile [5]. We previously reported the presence of GSCs that highly express CD44 and exhibit high invasiveness in the tumor periphery in patients with highly invasive GBM. Patients with this type of GBM exhibit earlier tumor progression and worse survival compared to patients with a less-invasive/ highly proliferative type of GBM expressing low amounts of CD44 in the tumor periphery [6]. However, it is unclear how such highly invasive GSCs change from a migratory to proliferative nature upon tumor recurrence and progression in GBM.

CD44 is a multifunctional cell surface adhesion receptor involved in regulating the progression, invasion, and metastasis of cancer cells [7, 8]. CD44 promotes tumor cell migration and invasion by binding to the specific ligand hyaluronic acid (HA) in various cancers, including GBM $[9,10]$. Other ligands in addition to HA, including collagen, matrix metalloproteinases, and osteopontin (OPN), also bind to CD44 [11-13]. Among these ligands, OPN is highly expressed in GBM and exhibits diverse functions related to proliferation, angiogenesis, tumorigenesis, and invasion [14-16]. OPN is reportedly overexpressed in GSCs and participates in maintaining stemness and activating the epidermal growth factor receptor (EGFR) [17]. In addition, Pietras et al. reported that interaction between OPN and CD44 promotes glioma stem cell-like phenotypes and aggressive glioma growth in vivo via enhanced hypoxia-inducible factor (HIF)-2a activity [18]. That is, CD44 may be regarded as a dual-function molecule that promotes both the migration and proliferation of cancer stem cells in response to binding to specific ligands.

GSCs are thought to be located in a hypoxic microenvironment called the "niche," where the stemness of the GSCs is maintained. The invasion area of the tumor periphery in GBM is characterized by a lack of neovascularization and disruption of the blood-brain barrier. This hypoxic environment can generate a tumor-border niche for GSCs. Hypoxia activates HIF genes, including the genes encoding HIF-1a and HIF$2 a[19,20]$. HIF-1 $a$ and HIF-2 $a$ are structurally similar, but differ in the several aspects including requirement of oxygen for their activation, target genes and expressing cells [21-23].

Our previous study demonstrated that HIF-1 $\mathrm{a}$ and CD44 are expressed at high levels in the tumor periphery in highly invasive GBM; in low-invasiveness/highly proliferative GBM, HIF-2a is expressed at higher levels and CD44 at lower levels in the tumor periphery compared with highly invasive GBM [6]. In low-invasiveness/highly proliferative GBM, higher amounts of vascular endothelial growth factor (VFGF) 
are expressed in the tumor periphery compared with highly invasive/low-proliferation GBM. These results suggest that differences in the level of hypoxia in the GSC microenvironment at the tumor periphery have a critical effect on the invasiveness and proliferation activity of GBM tumors through activation of HIF-1a and HIF-2a.

In the present study, we examined the effects of hypoxia on the expression and functions of HIFs, CD44 and OPN in order to elucidate the role of these molecules in GBM tumor invasion and proliferation. In addition, we investigated the effect of hypoxia on the functional role resulting from interaction between CD44 and OPN. We also investigated the anti-tumor effects of CD44 knockdown in vitro and in vivo using a GSC-transplantation mouse model. Identifying a key molecule closely related to the transition from a highly invasive phenotype to low-invasiveness/highly proliferative phenotype would provide an effective therapeutic target for both primary and recurrent GBM.

\section{Methods}

\section{GSC culture and hypoxic treatment}

Two human GSC lines, GSL-1 and GSL-2, were used in the present study. These cell lines were previously established from the primary cell culture of tissues surgically obtained from the tumor periphery of GBM patients [6]. The stemness of these GSC lines (previously designated SFC-1 and SFC-2) was confirmed by evaluating their sphere-forming ability, and the lines were then renamed GSL-1 and GSL-2, respectively.

Cells were cultured in serum-free DMEM/Ham's F-12 medium (Wako) containing $10 \mu \mathrm{g} / \mathrm{ml}$ insulin (Wako), $10 \mathrm{nmol} / \mathrm{I}$ recombinant human basic fibroblast growth factor, $10 \mathrm{nmol} / \mathrm{I}$ recombinant human epidermal growth factor, $5 \mu \mathrm{mol} / \mathrm{I}$ heparin, N2 supplement (Wako), GlutaMAX supplement (GIBCO), and penicillin/streptomycin/amphotericin B mixture (neural stem cell medium: NSCM). Growth factors were purchased from PeproTech (London, UK). The cells were maintained at $37^{\circ} \mathrm{C}$ under humidified $5 \%$ $\mathrm{CO}_{2} / 95 \%$ air atmosphere as the normoxic condition $\left(21 \% \mathrm{O}_{2}\right)$. For severe hypoxia treatment, the cells were incubated for $3 \mathrm{~h}$ in an atmosphere of $1 \% \mathrm{O}_{2}, 5 \% \mathrm{CO}_{2}$, and $94 \% \mathrm{~N}_{2}$ in a multi-gas incubator (APM50D, ASTEC). For moderate hypoxia treatment, the cells were incubated for $6 \mathrm{~h}$ in an atmosphere of $5 \%$ $\mathrm{O}_{2}, 5 \% \mathrm{CO}_{2}$, and $90 \% \mathrm{~N}_{2}$.

For sphere formation assay, $1 \times 10^{6}$ cells were seeded onto $10 \mathrm{~cm}$ culture dish and incubated for 7 days in the NSCM at normoxia. Then, the cells were sprit in six-well suspension culture plates at a density of 1000 cells/well in $2 \mathrm{ml} \mathrm{NSCM}$. After 7 days, formation of spheres was confirmed and the number was counted.

\section{Treatment of cells with small interfering RNA (siRNA)}

The sequences of siRNAs for HIF-1a, HIF-2a, CD44, and OPN are listed in Table S1. As a control for each siRNA, we used a corresponding random siRNA sequence (5'-GCGCGCUUUGUAGGAUUCG dTdT-3'). GSCs were transfected with each siRNA using Lipofectamine 3000 reagent (Invitrogen, Grand Island, NY, USA) 
according to the manufacturer's instructions. After a 24-h incubation of GSCs transfected with each siRNA, the culture medium was changed to remove the Lipofectamine, and subsequent experimentation was performed.

\section{Establishment of stable CD44-knockdown cells}

Lentiviral particles were generated using the shRNA expression vector pLK0.1-puro, which carries a shRNA sequence against CD44 (CD44 MISSION shRNA, SHCLNG-NM_000610, Sigma Aldrich) together with the MISSION Lentiviral packaging mix (SHP001, Sigma Aldrich), according to the manufacturer's instructions. Briefly, HEK 293T cells were co-transfected with the two products described above using Lipofectamine 3000 reagent (Invitrogen). The supernatant containing virus particles was harvested $48 \mathrm{~h}$ after transfection and used to infect GSCs. After $48 \mathrm{~h}$ of incubation, infected cells were selected using puromycin $(0.5 \mu \mathrm{g} / \mathrm{ml}$; Invitrogen).

\section{RNA isolation and quantitative real-time-polymerase chain reaction (qRT-PCR)}

Total RNA was extracted from GSCs and tissue of each tumor sample (core and periphery) using ISOGEN (Nippon Gene, Tokyo, Japan) according to the manufacturer's instructions. cDNA was synthesized using ReverTra Ace qPCR RT Master Mix with a gDNA remover kit (Toyobo). qPCR analysis was performed using Fast Start Universal SYBR Green Master Mix (Roche Diagnostic Japan) with an MJ mini instrument (BioRad, Hercules, CA, USA). All gene-specific mRNA expression values were normalized relative to the expression level of the housekeeping (reference) gene, glyceraldehyde-3-phosphate dehydrogenase (GAPDH). Quantification of gene expression was performed using $\Delta \mathrm{Ct}$ values, wherein $\Delta \mathrm{Ct}$ is defined as the difference between the target and reference gene Ct values. All primer sequences are listed in Table S2.

\section{Western blot analysis}

Cells grown on poly-L-lysine-coated dishes were lysed using RIPA buffer solution. The lysates were electrophoresed, transferred onto nitrocellulose membranes, and immunoblotted with antibodies to $\beta$ actin (1:1000; mouse monoclonal; Sigma), HIF-1a (1:250; mouse monoclonal; BD Biosciences), HIF-2a (1:250; rabbit polyclonal; abcam), OPN (1/200, rabbit polyclonal, abcam) or CD44 (1:250; mouse monoclonal; Cell Signaling Technology). Following incubation with alkaline phosphatase-conjugated secondary antibody (Promega), immunoreactions were developed using nitro blue tetrazolium and 5bromo-4-chloro-3-indolyl phosphate.

\section{Immunohistochemical analysis}

Freshly dissected tissue blocks surgically obtained from the tumor periphery of GBM patients were frozen and sliced into $5-\mu \mathrm{m}$-thick sections. These sections were stored at $-80^{\circ} \mathrm{C}$ for later use. The sections were permeabilized and blocked with Tris-buffered saline containing $0.1 \%$ Tween 20 (TBSt) and $1 \mathrm{mg} / \mathrm{ml}$ bovine serum albumin (BSA-TBSt) for $30 \mathrm{~min}$. For double-labeling immunofluorescence, sections were incubated in a humidified chamber overnight at $4^{\circ} \mathrm{C}$ with a mixture of two primary antibodies diluted in 
BSA-TBSt, one antibody to OPN (1/200, rabbit polyclonal, abcam; $1 / 100$, mouse monoclonal, R\&D) and the other to Nestin (1/200, mouse monoclonal, Chemicon International). After washing with TBSt, sections were treated with DyLight 488-labeled anti-mouse and Cy3-conjugated anti-rabbit IgG secondary antibodies (1:1000, Jackson ImmunoResearch, West Grove, PA, USA). Hoechst 33342 (Sigma-Aldrich) was used for nuclear staining. The immunostained specimens were observed under a conventional microscope (BX52; Olympus, Tokyo, Japan).

Cells were fixed with $4 \%$ paraformaldehyde (PFA), permeabilized, and blocked with BSA-TBSt for 30 min. The cells were then incubated in a humidified chamber overnight at $4^{\circ} \mathrm{C}$ with a mixture of two primary antibodies diluted in BSA-TBSt: antibodies to CD44 (1:250, mouse monoclonal, Cell Signaling Technology) and HIF-1a (1/250, rabbit polyclonal, Novus) or HIF-2a (1/100, rabbit polyclonal, abcam) and OPN (1/250, mouse monoclonal, abcam). The cells were then treated according to the steps described above.

Sections of mouse brain were deparaffinized in Histo-Clear (Cosmo Bio), hydrated in a graded alcohol series and subjected to heat-activated antigen retrieval. After blocking endogenous peroxidase activity, the sections were incubated in a humidified chamber overnight at $4^{\circ} \mathrm{C}$ with monoclonal antibodies to CD44 (1:200, Cell Signaling Technology), Ki-67 (1:200, Dako), Nestin (1/200, Chemicon International), or Sox2 (1/200, R\&D Systems) diluted in BSA-TBSt. Subsequently, the sections were washed with TBSt and incubated with biotinylated secondary antibody for $1 \mathrm{~h}$ at room temperature. The reaction complexes were stained with diaminobenzidine and counterstained with hematoxylin.

\section{Measurement of OPN secreted out of GSCs}

When GSCs reached confluence, the culture medium was removed, and the cells were washed with PBS. After addition of serum-free, low-glucose DMEM, the cells were cultured for $24 \mathrm{~h}$. The cells were then centrifuged, and the supernatant was used as conditioned medium. The concentration of OPN in the conditioned medium was determined using an OPN ELISA kit (Sigma-Aldrich) according to the manufacturer's protocol.

\section{Cell invasion and migration assays}

The invasiveness of cultured GSCs was assessed with an in vitro assay using Falcon cell culture inserts (Becton Dickinson Biosciences, CA, USA) and a reconstituted basement membrane, Matrigel (Becton Dickinson Biosciences), as previously described [24,25]. Briefly, GSCs were suspended in DMEM containing $0.1 \%$ BSA and seeded onto the insert filters at a density of $5 \times 10^{4}$ cells/insert. The insert was placed in the lower wells of the Falcon 24-well plate containing $500 \mu \mathrm{l}$ of DMEM with $1 \%$ FBS and incubated for $24 \mathrm{~h}$ at $37^{\circ} \mathrm{C}$ under normoxic conditions or $1 \% \mathrm{O}_{2}$ hypoxic conditions. GSC migration was assayed using the modified Boyden chamber method with 48-well microchemotaxis chambers (Nucleopore, Pleasanton, CA, USA), as previously described [26,27]. GSCs in DMEM containing $0.1 \%$ BSA (at a density of $1 \times 10^{4}$ cells $/ \mathrm{ml}$ ) were placed in the upper well, and DMEM containing $1 \%$ FBS was placed in the lower well. A polyvinylpyrolidone-free polycarbonate membrane with $8-\mu \mathrm{m}$ pores (EMD Millipore, 
Bedford, MA, USA) was used. To examine the effect of OPN on CD44-induced migration and invasion, 1 $\mu \mathrm{g} / \mathrm{ml}$ of OPN (Sigma Aldrich) was added to the upper well. To examine the effect of OPN addition on HA (Wako, Japan)-stimulated migration and invasion of GSCs, HA was added to the lower well at a concentration of $0.5 \mathrm{mg} / \mathrm{ml}$. The chamber was incubated for $6 \mathrm{~h}$ at $37^{\circ} \mathrm{C}$ under normoxic conditions or $1 \%$ or $5 \% \mathrm{O}_{2}$ hypoxic conditions. In both assays, cells on the upper membrane surface were mechanically removed. Cells that had invaded or migrated to the lower side of the membrane were fixed, stained with $0.1 \%$ crystal violet, and examined under a microscope $(\times 400)$ to determine the number of cells in three random fields.

\section{In vivo xenograft experiments}

Control and CD44-knockdown cells $\left(1 \times 10^{6}\right)$ were suspended in $5 \mu$ of Matrigel and injected into the brain of 6-week-old male NOD/SCID mice purchased from CLEA Japan, Inc. ( $\mathrm{n}=6$ mice per both group). that had been anesthetized intraperitoneally with a mixture of medetomidine $(0.75 \mathrm{mg} / \mathrm{kg})$, midazolam $(4 \mathrm{mg} / \mathrm{kg})$, and butorphanol tartrate $(5 \mathrm{mg} / \mathrm{kg})$. MRI was performed to confirm tumorigenesis before the mice were euthanized by decapitation. The brain of each mouse was dissected and fixed in $4 \%$ PFA at $4^{\circ} \mathrm{C}$ overnight. After fixation, the brains were embedded in paraffin, sliced into 5 - $\mu \mathrm{m}$-thick sections, and stained with hematoxylin and eosin. The duration from cell injection to decapitation was evaluated as survival time.

\section{Statistical analysis}

Values are expressed as the mean \pm standard deviation (SD), and the data were compared using the Student's t-test (unpaired). Kaplan-Meier plots were generated to estimate unadjusted time-to-event variables. The log-rank test was performed to assess the statistical significance of differences between groups. Significance was set at $P<0.05$.

\section{Results}

\section{Effect of hypoxia on the expression of HIFs, CD44, and OPN in GSCs}

To assess the expression patterns of HIFs (HIF-1a and HIF-2a), CD44, and OPN in the two GSC lines (GSL1 and GSL-2) under different levels of hypoxia, the mRNA expression of these molecules was examined under normoxic $\left(21 \% \mathrm{O}_{2}\right)$ and hypoxic conditions $\left(1 \% \mathrm{O}_{2}\right.$ [severe] and $5 \% \mathrm{O}_{2}$ [moderate]). Expression of HIF-1a and CD 44 mRNAs was up-regulated to a greater degree under the $1 \% \mathrm{O}_{2}$ condition than under normoxic or moderately hypoxic $\left(5 \% \mathrm{O}_{2}\right)$ conditions in both GSC lines (Fig. 1). In contrast, the expression of HIF-2 $a$ and OPN mRNAs was more strongly up-regulated in both GSC lines under moderate hypoxia than under conditions of normoxia or severe hypoxia (Fig. 1).

\section{HIF-1a up-regulates CD44 expression in GSCs under severe hypoxia}


We investigated whether HIF-1a affects the expression of CD44 under the condition of severe hypoxia (1\% $\mathrm{O}_{2}$ ). Silencing of the HIF-1 a gene using HIF-1a siRNA resulted in significant suppression of both the mRNA and protein expression of CD44 in both GSC lines under the condition of severe hypoxia (Fig. 2A, B). Double-labeling immunofluorescence demonstrated that CD44 and HIF-1a co-localized in GSCs subjected to severe hypoxia (Fig. 2C).

\section{HIF-2a up-regulates OPN expression in GSCs under mild hypoxia}

We also investigated whether HIF-2a affects OPN expression by GSCs cultured under moderate hypoxia $\left(5 \% \mathrm{O}_{2}\right)$. Silencing of the HIF-2a gene using HIF-2a siRNA resulted in significant suppression of both the mRNA and protein expressions of OPN in GSCs cultured in the presence of $5 \% \mathrm{O}_{2}$ (Fig. 2D, E). In addition, double-labeling immunofluorescence demonstrated the co-localization of OPN and CD44 in both GSC lines, indicating that OPN binds to CD44-expressing GSCs, regardless of the level of CD44 expression (Fig. 2F).

\section{Gene and histologic expression of OPN in GBM tissues and extracellular secretion of OPN in GSCs}

We examined the expression pattern of OPN in human GBM tissues and examined whether GSCs secrete OPN. The expression of OPN mRNA was examined in the core and peripheral tissues of two resected GBM tumors. One of these tumors was a highly invasive type that expressed a high level of CD44 (HItumor), and the other tumor was a low-invasiveness/highly proliferative type that expressed CD44 at a low level and VEGF at a high level (LI-tumor). Natures of these two types of GBMs were described in the supplementary figure (Supplement Fig. 2). The LI-tumor expressed a significantly higher level of OPN in the tumor periphery than the HI-tumor (Fig. 3A). Double-labeling immunofluorescence demonstrated that OPN co-localized with Nestin in the peripheral tissues of both tumor types (Fig. 3B). In addition, OPN colocalization with nestin was more pronounced in the LI-tumor than HI-tumor. We confirmed that both GSC lines secreted OPN in proportion to the level of OPN mRNA and protein expression. The concentration of OPN in the culture medium of cells incubated under the normoxic condition was much higher in medium of GSL-2 cells than GSL-1 cells (Fig. 3C, D).

\section{Severe hypoxia enhances GSC migration and invasion, and CD44 knockdown inhibits hypoxia-induced GSC migration and invasion}

In vitro migration and invasion assays revealed that the migration and invasion of both GSC lines was markedly enhanced under the severe hypoxia condition of $1 \% \mathrm{O}_{2}$ compared with normoxia (Fig. 4A). In contrast, moderate hypoxia $\left(5 \% \mathrm{O}_{2}\right)$ had almost no effect on GSC migration and invasion. The enhancement of GSC invasion and migration induced by severe hypoxia was significantly inhibited by knockdown of the CD44 gene using CD44 siRNA cells cultured in the presence of $1 \% \mathrm{O}_{2}$ (Fig. 4A).

\section{OPN inhibits GSC migration and invasion}


OPN inhibited CD44- mediated migration and invasion in both GSC lines (Fig. 4B). To investigate the inhibitory effects of OPN on CD44-promoted migration and invasion of GSC lines, we performed migration and invasion assays by adding HA to the lower chamber wells. Addition of HA to the lower wells of the chamber increased the migration and invasion behaviors of both GSC lines. OPN inhibited the HA-stimulated migration and invasion of GSC lines in a dose-dependent manner, and the inhibitory effect was not decreased compared with migration and invasion of GSC lines without HA (Fig. 4B). These results thus suggest that OPN may exerts an inhibitory effect on migration and invasion of GSCs before the cells adhere to the membrane filter placed in the bottom of the upper well of Boyden chamber.

\section{Effects of CD44 knockdown and OPN silencing on GSC proliferation}

Among five clones of bacteria maintained in glycerol stocks, clone 2 most-effectively inhibited CD44 expression in both GSC lines (Supplement Fig. 1); therefore, we used this clone to generate CD44knockdown cells in the following experiments. Knockdown of the CD44 gene significantly reduced the expression of other stem cell markers in both GSC lines, including CD133, Nestin, Oct3/4, and Sox2, in addition to the angiogenesis factor VEGF (Fig. 5A). In contrast, knockdown of CD44 did not affect the proliferation of either GSC line (Fig. 5B). Silencing OPN using OPN siRNA resulted in decreased expression of CD44 and Nestin but increased expression of VEGF (VEGF expression was significantly increased only in GSL-2 cells) (Fig. 5C). Incubation of GSCs under the moderate hypoxia condition (5\% $\mathrm{O}_{2}$ ) markedly increased the cell growth rate, whereas culture under the severe hypoxia condition $\left(1 \% \mathrm{O}_{2}\right)$ resulted in a decreased growth rate (Fig. 5D). The enhancement in growth rate under moderate hypoxia of $5 \% \mathrm{O}_{2}$ was significantly suppressed by reducing OPN expression to a lower level, as compared with the growth rate under the condition of normoxia.

\section{In vivo study using a CD44-knockdown transplanted-GSC mouse model}

The effect of CD44 knockdown on various histologic features of tumor tissues in mouse brain and the survival of these mice were investigated using NOD/SCID mice receiving transplanted GSCs with and without CD 44 knockdown. Tumors generated by GSC transplantation with CD 44 knockdown exhibited less migration of tumor cells around the tumor mass and exhibited a well-demarcated boundary compared to controls (Fig. 6A). The proliferative Ki-67 staining index of tumors from mice with transplanted GSCs with CD44 knockdown was not significantly different from that of the control (maximum Ki-67 LI: 50.7\% [former] vs. 50.9\% [latter]; P=0.973). In contrast, positive expression of the stem cell markers Nestin and Sox 2 was observed in tumors generated by transplantation of GSCs without CD44 knockdown, whereas no expression of these markers was observed in tumors generated by transplantation of GSCs with CD44 knockdown (Fig. 6B). As a result, mice bearing GSC-induced tumors with CD44 knockdown survived for significantly longer than tumor-bearing mice without CD44 knockdown (Fig. 6C).

\section{Discussion}


In the present study, we demonstrated that the highly invasive phenotype of GSCs expressing high levels of CD44 can be converted to a low-invasiveness/highly proliferative phenotype by switching from severe to moderate hypoxia. The severity of hypoxia necessary to affect the activation of HIF-1a versus HIF-2a differed in two GSC lines examined in this study. HIF-1 a expression was up-regulated by severe hypoxia ( $1 \%$ 02) but not affected by moderate hypoxia ( $5 \%$ 02). In contrast, under moderate hypoxia, HIF-2a expression was significantly up-regulated to a much higher level than under severe hypoxia. Following upregulation, HIF-1a activated the expression of CD44 under the severe hypoxia condition (HIF-1 a/CD44 pathway), and the up-regulated expression of CD44 induced a highly invasive phenotype in the GSCs. Elevated HIF-2a expression significantly up-regulated the expression of OPN (HIF-2a/OPN pathway). Thus, tumors exhibiting high OPN expression and low CD44 expression may represent GSCs with a low invasiveness/highly proliferative phenotype. These relationships between CD44 and OPN in the phenotype of GBM may be supported by the results that another GSC line (GDC40) (glioblastoma-derived cells; kindly provided by Dr. Y. Kanemura, Osaka National Hospital, Japan) [28], which expressed much lower CD44 and higher VEGF levels than the GSL-1 and GSL-2 lines, showed high expression of OPN and exhibited low migration and invasion both in vitro and in vivo. (Supplement Fig. 3)

CD44 is a major cellular adhesion molecule for HA, an extracellular matrix component [29]. CD44 also interacts with a variety of other ligands and plays a crucial role in many cellular processes [10, 29]. These other ligands include OPN, a multifunctional glycophosphoprotein that plays a critical role in the maintenance of the stemness and tumorigenicity of GSCs [30-32]. To elucidate the relationship between CD44 and OPN in the progression of highly invasive GBM tumors, in the present study, we examined the expression of OPN and the functions of CD44 and OPN under severe $\left(1 \% \mathrm{O}_{2}\right)$ and moderate $\left(5 \% \mathrm{O}_{2}\right)$ hypoxia. In GBM tumor tissues, we found that OPN was expressed at considerably higher levels in the peripheral tissues of low-invasiveness/highly proliferative tumors. However, such high expression of OPN in the tumor periphery was not commonly observed in highly invasive GBM tumors. Several studies have reported that OPN expression in gliomas varies and that the OPN expression level is correlated with glioma grade; thus, high OPN expression is correlated with poor prognosis in GBM patients [33, 34]. Friedmann-Morvinski et al. reported that OPN is expressed at higher levels in the border area of GBM tumors compared with the central area and that the invasive and more-aggressive tumor cells located along the periphery of the tumors express high levels of OPN [32]. Our results were in agreement in terms of the location of tumor cells expressing high levels of OPN in low-invasiveness/demarcated-type GBM tumors. However, in our study, OPN expression was not as high at the periphery of highly invasive GBM tumors.

We previously reported that CD44 markedly increases GSC migration and invasion under normoxic conditions [6]. In the present study, severe hypoxia $\left(1 \% \mathrm{O}_{2}\right)$ further enhanced GSC migration and invasion compared with the normoxic condition, and both the migration and invasiveness of GSCs were inhibited by silencing CD44 expression using siRNA. These results indicate that under conditions of severe hypoxia, activated CD44 (HIF1a/CD44 pathway) promotes the migratory and invasive activities of GSCs residing in the tumor periphery, resulting in the highly invasive phenotype of GBM. 
We also demonstrated that OPN inhibits GSC migration and invasion under the normoxic condition. To elucidate the mechanism of the inhibitory activity of OPN, we investigated the effect of OPN on HAinduced GSC migration and invasion. The inhibitory effect of OPN on HA-stimulated migration and invasion of GSCs was observed to the same degree as CD44-promoted migration and invasion of GSCs without $\mathrm{HA}$ and the effect was enhanced in an OPN dose-dependent manner. The mechanism underlying the inhibition of GSC migration and invasion by OPN is not clear. However, it can be hypothesized that OPN bound to CD44 on the GSCs regulates a signal pathway to promoting cell motility before the cells adhere to the substrates, resulting in inhibition of migration and invasion in GSCs.

OPN has a variety of known functions, particularly in regard to tumor progression and metastasis. An important question then is how does the inhibition of CD44-induced GSC migration and invasion due to the interaction of OPN and CD44 affect tumor progression? To answer this question, we investigated the roles of CD44 and OPN on GSC proliferation under hypoxic conditions. Knockdown of CD44 did not affect tumor proliferation in the in vitro study. In contrast, the growth rate of GSCs was significantly enhanced under the condition of moderate hypoxia $\left(5 \% \mathrm{O}_{2}\right)$, but this effect was suppressed by silencing OPN using siRNA. These results indicate that CD44 does not affect the proliferation of GSCs, whereas OPN promotes GSC proliferation under moderately hypoxic conditions.

It has been reported that binding of OPN to CD44 induces the intramembranous cleavage of CD44 via presenillin-dependent $y$-secretase $[18,35,36]$, resulting in the release of the CD44 intracellular domain (CD44-ICD) into the cytoplasm. Cytoplasmic CD44-ICD translocates to the nucleus and activates the transcription of several genes involved in cell survival, inflammation, tumor growth, and tumor invasion $[36,37]$. The CD44-ICD also stimulates HIF-2a activity, resulting in increased stemness of tumor cells, which promotes aggressive tumor growth in vivo and enhances the radiation resistance of tumors [18, 38]. Interaction between OPN and CD44 also activates the EGFR pathway, leading to cell proliferation [17].

The results described above may explain the inhibition of CD44-induced GSC migration and invasion by OPN. In general, tumor cells do not proliferate when they move, and vice versa. This phenomenon is known as the "migration/proliferation dichotomy" theory, or the "go or grow mechanism" [39-41]. GBM is a highly invasive tumor, and the GSCs of these tumors exhibit high migratory activity, but when these tumor recur, they must shift from migration to proliferation in order to generate tumor mass [42]. The dual functionality of CD44 could play a critical role in the phenotypic transition that is thought to be required for GBM tumor recurrence and progression. Consequently, the functional linkage between CD 44 and OPN regulated by differing degrees of hypoxia could possibly be exploited as a therapeutic target in the treatment of not only primary GBM but also recurrent GBM.

These hypoxia-related molecular events suggest that the level of hypoxia in the microenvironment surrounding GSCs residing in the tumor periphery niche determines the GBM phenotype. In other words, the GBM phenotype can be converted by changing the degree of hypoxia in the microenvironment. Considering applications to clinical practice, in a severely hypoxic environment $\left(\sim 1 \% \mathrm{O}_{2}\right)$, such as that of perinecrotic and highly invasive areas where there is almost no neovasculature, GSCs up-regulate CD44 
expression via HIF-1a activation (HIF-1a/CD44 pathway), thus yielding tumor stem cells with a high migratory and invasive nature. That is, CD44 may play the role of an accelerator that promotes GSC migration and invasion. In contrast, in a moderately hypoxic environment $\left(3 \sim 5 \% \mathrm{O}_{2}\right)$, such as perivascular and tumor-border areas with low vascularity, GSCs up-regulate OPN expression via HIF-2a activation (HIF-2a/OPN pathway); OPN then binds to CD44, resulting in the release of the CD44-ICD by intramembranous cleavage. This reaction process may inhibit CD44-induced GSC migration and invasion. The released CD44-ICD activates the transcription of various genes that have multiple effects in GSCs, including increasing their stemness, proliferation rate, and radio-resistance. That is, OPN may function as the modulator by which CD44 executes multiple functions (Fig. 7). Our data suggest that inhibition of CD44 expression may be an effective option for the treatment of highly invasive primary GBM tumors, whereas inhibition of OPN expression may be an effective option for the treatment of lowinvasiveness/highly proliferative primary and recurrent GBM tumors. Lamour et al. reported that OPN has major effects on GSC stemness and tumorigenicity and suggested that OPN suppression would be an effective therapy for stopping the progression of GBM tumors [15].

One of the most significant factors that makes it difficult to treat GBM tumors is their highly invasive nature, which makes it impossible to completely resect these tumors. If the tumors could be rendered less invasive, they could be almost totally resected when they are not located in eloquent areas of the brain. Indeed, patients with low-invasiveness/highly proliferative GBM tumors that express CD44 at a low level in the tumor periphery exhibit much longer progression-free and overall survival than those with highly invasive GBM tumors expressing high levels of CD44 [6]. In addition, OPN was much more highly expressed in the tumor periphery in patients with low-invasive/highly proliferative GBM than those with highly invasive GBM. Consequently, it may be possible to develop a novel and highly effective therapy for GBM by targeting a key molecule that regulates the transition from an invasive to less-invasive GSC phenotype. We therefore focused on CD44 and OPN as potential therapeutic targets to identify an optimal strategy for treating GBM. CD44 knockdown in GSCs significantly decreased the mRNA expression of various stem cell markers (CD133, Nestin, Oct3/4, and Sox2) compared with the control. Lamour et al. reported that OPN knockdown decreased the expression of Sox2, Oct3/4, and Nanog in glioma-initiating cells by suppressing the release of CD44-ICD fragments, which function in activating the expression of these stem cell markers [15]. Decreased expression of stem cell markers via CD44 knockdown may also have involved a reduction in intracellular CD44-ICD levels due to the reduced availability of CD44 for binding to OPN.

GSC invasion was markedly inhibited by knockdown of CD44 both in vitro and in the in vivo mouse xenograft model. In contrast, CD44-knockdown GSCs proliferated the almost same high rate as the parent cells in vitro, but the growth rate of brain tumors generated in mice by transplantation of CD44knockdown GSCs was strongly suppressed, resulting in a significantly longer survival time of these mice compared with the control. PCR analyses demonstrated that the expression of VEGF mRNA in CD44knockdown cells was markedly decreased compared with the control, but the Ki-67 LI of tumor tissue generated from CD44-knockdown GSCs was not significantly decreased compared with the control. 
These findings suggest that the inhibition of tumor growth in the mice brains might be caused by reduction of GSCs' stemness via CD44 knockdown rather than suppression of angiogenesis due to decreased VEGF expression.

Although silencing OPN expression in GSCs reduced the expression of CD44 and Nestin, in another GSC line, GDC40 silencing of OPN resulted in increased CD44 expression and decreased VEGF expression (Supplement Fig. 3). The GDC40 line, which presents low migration and invasion both in vitro and in vivo, exhibited a decrease in growth rate but induced diffuse infiltration of cells around tumor spheres after OPN silencing (Supplement Fig. 4). These results in GSCs with a different phenotype suggest that inhibition of OPN expression carries the risk of inducing the formation of more-invasive tumors. Taken together, these data suggest that inhibition of CD44 alone or in combination with OPN suppresses both the invasion and proliferation of GSCs in vivo and therefore could become an effective targeting therapy for treating not only highly invasive GBM but also low-invasiveness/highly proliferative GBM. A more precise elucidation of the molecular pathways involved in the phenotypic transition of GSCs would open the door to a new and more effective molecular-targeting therapy for patients with GBM.

\section{Conclusions}

The expression of CD44 in GSCs was up-regulated via activation of HIF-1a under the condition of severe hypoxia $\left(1 \% \mathrm{O}_{2}\right)$, inducing the cells to assume a highly invasive phenotype. In contrast, OPN expression in GSCs was up-regulated via HIF-2 $a$ activation under the condition of moderate hypoxia $\left(5 \% \mathrm{O}_{2}\right)$, inducing the cells to assume a less-invasive and highly proliferative phenotype. Up-regulated CD44 expression promoted the migration and invasion of GSCs under the severe hypoxia condition but did not affect the in vitro proliferation of GSCs. In contrast, secreted OPN bound to CD44 and inhibited the migration and invasion of GSCs while increasing their proliferation under the moderate hypoxia condition. Knockdown of CD44 expression in GSCs inhibited both cell migration/invasion and proliferation in an in vivo mouse xenograft model, thus resulting in much longer survival of mice with tumors generated from transplanted GSCs compared with the control. In the transition between the invasive and proliferative phenotypes of GSCs, CD44 plays a key role as an accelerator that promotes migration and invasion, whereas OPN may play a role as a multifunctional modulator via its interaction with CD44. Thus, a molecular therapy targeting CD44 alone or in combination with OPN could become an effective strategy for treating both primary and recurrent GBM.

\section{Abbreviations}

GBM: glioblastoma multiforme; GSC:glioma stem-like cell; OPN:osteopontin; GAPDH:glyceraldehyde-3phosphate dehydrogenase; TBSt:Tris-buffered saline containing $0.1 \%$ Tween 20; BSA:bovine serum albumin, SD:standard deviation

\section{Declarations}




\section{Ethics approval and consent to participate}

The study was approved by the Institutional Review Board of the Ethics Committee for Clinical Research of Ehime University Hospital.

\section{Consent for publication}

Not applicable.

\section{Availability of data and materials}

The data used to support the findings of the study are available from the corresponding author upon request.

\section{Competing interests}

The authors declare that there is no conflict of interest regarding the publication of this paper.

\section{Funding}

Not applicable.

\section{Authors' contributions}

$\mathrm{MN}$ and $\mathrm{Al}$ designed the research, performed the experiments, interpreted the results, and wrote the manuscript. TO and TK interpreted the results and helped write and revise the manuscript. HY and JT provided technical guidance for all experiments. SOz and YO assisted with some of the in vitro experiments. YK provided the GDC40 glioblastoma-derived cells. SM, SSu, SSh and HW helped interpret the results. SOh and SK performed surgery on the patients whose tumor tissues were used in this study. RK provided pathological analysis. All authors read and approved the final manuscript.

\section{Acknowledgements}

The authors would like to express their gratitude to Satsuki Myoga (Department of Pathology, Ehime University Hospital, Japan) and Takeshi Kiyoi (Advanced Research Support Center, Ehime University, Japan) for their assistance with acquisition of pathologic specimens.

\section{References}

1. Stupp R, Hegi ME, Mason WP, et al. Effects of radiotherapy with concomitant and adjuvant temozolomide versus radiotherapy alone on survival in glioblastoma in a randomised phase III study: 
5-year analysis of the EORTC-NCIC trial. Lancet Oncol. 2009;10:459-66.

2. Giese A, Kucinski T, Knopp U, et al. Pattern of recurrence following local chemotherapy with biodegradable carmustine (BCNU) implants in patients with glioblastoma. J Neurooncol. 2004;66:351-60.

3. Oh J, Sahgal A, Sanghera P, et al. Glioblastoma: patterns of recurrence and efficacy of salvage treatments. Can J Neurol Sci. 2011;38:621-5.

4. Munthe S, Petterson SA, Dahlrot RH, et al. Glioma cells in the tumor periphery have a stem cell phenotype. PLoS One. 2016;11:e155106.

5. Angelucci C, D’Alessio A, Lama G, et al. Cancer stem cells from peritumoral tissue of glioblastoma multiforme: the possible missing link between tumor development and progression. Oncotarget. 2018;9:28116-130.

6. Nishikawa M, Inoue A, Ohnishi T, et al. Significance of glioma stem-like cells in the tumor periphery that express high levels of CD44 in tumor invasion, early progression, and poor prognosis in glioblastoma. Stem Cells Int. 2018. doi:10.1155/2018/5387041.

7. Naor D, Nedvetzkis S, Golan I, et al. CD44 in cancer. Crit Rev Clin Lab Sci. 2002;39:527-79.

8. Lesley J, Hyman R. CD44 structure and function. Front Biosci. 1998;3:D616-30.

9. Aruffo A, Stamenkovic I, Melnick M, et al. CD44 is the principal cell surface receptor for hyaluronate. Cell. 1990;61:1303-13.

10. Bourguignon LY, Zhu H, Shao L, et al. CD44 interaction with c-Src kinase promotes cortactin-mediated cytoskeleton function and hyaluronic acid-dependent ovarian tumor cell migration. J Biol Chem. 2001;276:7327-36.

11. Goodison S, Urquidi V, Tarin D. CD44 cell adhesion molecules. Mol Pathol. 1999;52:189-96.

12. Merzak A, Koocheckpour S, Pilkington GJ. CD44 mediates human glioma cell adhesion and invasion in vitro. Cancer Res. 1994;54:3988-92.

13. Yu Q, Stamenkovic I. Localization of matrix metalloproteinase 9 to the cell surface provides a mechanism for CD44-mediated tumor invasion. Genes Dev. 1999;13:35-48.

14. Rangaswami H, Bulbule A, Kundu GC. Osteopontin: role in cell signaling and cancer progression. Trends Cell Biol. 2006;16:79-87.

15. Matusan-Ilijas K, Behrem S, Jonjic N, et al. Osteopontin expression correlates with angiogenesis and survival in malignant astrocytoma. Pathol Oncol Res. 2008;14:293-8.

16. Jan HJ, Lee CC, Shih YL, et al. Osteopontin regulates human glioma cell invasiveness and tumor growth in mice. Neuro Oncol. 2010;12:58-70.

17. Lamour V, Henry A, Kroonen J, et al. Targeting osteopontin suppresses glioblastoma stem-like cell character and tumorigenicity in vivo. Int J Cancer. 2015;137:1047-57.

18. Pietras A, Katz AM, Ekström EJ, et al. Osteopontin-CD44 signaling in the glioma perivascular niche enhances cancer stem cell phenotypes and promotes aggressive tumor growth. Cell Stem Cell. 2014;14:357-69. 
19. Gordon JD, Simon MC. Hypoxia-inducible factors: central regulators of the tumor phenotype. Curr Opin Genet Dev. 2007;17:71-7.

20. Hu CJ, Wang LY, Chodosh LA, et al. Differential roles of hypoxia-inducible factor 1alpha (HIF-1alpha) and HIF-2alpha in hypoxic gene regulation. Mol Cell Biol. 2003;23:9361-74.

21. Kaur B, Khwaja EW, Severson EA, et al. Hypoxia and hypoxia-inducible-factor pathway in glioma growth and angiogenesis. Neuro-Oncol. 2005;7(2):134-53.

22. Li Z, Bao S, Wu Q, et al. Hypoxia-inducible factors regulate tumorigenic capacity of glioma stem cells. Cancer Cell. 2009;15:501-13.

23. Covello KL, Kehler J. Yu H, et al. HIF-2alpha regulates Oct-4: effects of hypoxia on stem cell function, embryonic development, and tumor growth. Genes Dev. 2006;20:557-70.

24. Albini A, Iwamoto $\mathrm{Y}$, Kleinman $\mathrm{HK}$, et al. A rapid in vitro assay for quantitating the invasive potential of tumor cells. Cancer Res. 1987;47:3239-45.

25. Ohnishi T, Arita N, Hayakawa T, et al. Purification of motility factor (GMF) from human malignant glioma cells and its biological significance in tumor invasion. Biochem Biophys Res Commun. 1993;193:518-25.

26. Ohnishi T, Arita A, Hayakawa T, et al. Motility factor produced by malignant glioma cells: role in tumor invasion. J Neurosurg. 1990;73:881-8.

27. Ohnishi T, Arita N, Hiraga S, et al. Fibronectin-mediated cell migration promotes glioma cell invasion through chemokinetic activity. Clin Exp Metastasis. 1997;15:538-46.

28. Bamba Y, Shofuda T, Kanematsu D, Nonaka M, Yamasaki M, Okano H, et al. Differentiation, polarization, and migration of human induced pluripotent stem cell-derived neural progenitor cells cocultured with a human glial cell line with radial glial-like characteristics. Biochem Bioph Res Co. 2014;447:683-88.

29. Senbanjo LT, Chellaiah MA. CD44: a multifunctional cell surface adhesion receptor is a regulator of progression and metastasis of cancer cells. Front Cell Dev Biol. 2017. doi:10.3389/fcell.2017.00018.

30. Denhardt DT, Noda M, O'Regan AW, et al. Osteopontin as a means to cope with environmental insults: regulation of inflammation, tissue remodeling, and cell survival. J Clin Invest. 2001;107:1055-61.

31. Wai PY, Kuo PC. The role of osteopontin in tumor metastasis. J Surg Res. 2004;121:228-41.

32. Friedmann-Morvinski D, Bhargava V, Gupta S, et al. Identification of therapeutic targets for glioblastoma by network analysis. Oncogene. 2016;35:608-20.

33. Bache $\mathrm{M}$, Kappler $\mathrm{M}$, Wichmann $\mathrm{H}$, et al. Elevated tumor and serum levels of the hypoxia-associated protein osteopontin are associated with prognosis for soft tissue sarcoma patients. BMC Cancer. 2010. doi:10.1186/1471-2407-10-132.

34. Sreekanthreddy P, Srinivasan H, Kumar DM, et al. Identification of potential serum biomarkers of glioblastoma: serum osteopontin levels correlate with poor prognosis. Cancer Epidemiol Biomarkers Prev. 2010;19:1409-22. 
35. Nagano O, Saya H. Mechanism and biological significance of CD44 cleavage. Cancer Sci. 2004;95:930-5.

36. Okamoto I, Kawano Y, Murakami D, et al. Proteolytic release of CD44 intracellular domain and its role in the CD44 signaling pathway. J cell Biol. 2001;155:755-62.

37. Miletti-Gonzalez KE, Murphy K, Kumaran MN, et al. Identification of function for CD44 intracytoplasmic domain (CD44-ICD): modulation of matrix metalloproteinase 9 (MMP-9) transcription via novel promoter response element. J Biol Chem. 2012;287:18995-9007.

38. Johansson E, Grassi ES, Pantazopoulou V, et al. CD44 interacts with HIF-2a to modulate the hypoxic phenotype of perinecrotic and perivascular glioma cells. Cell Rep. 2017;20:1641-53.

39. Hatzikirou H, Basanta D, Simon M, et al. 'Go or grow': the key to the emergence of invasion in tumour progression? Math Med Biol. 2012;29:49-65.

40. Giese A, Loo MA, Tran N, et al. Dichotomy of astrocytoma migration and proliferation. Int J Cancer. 1996;67:275-82.

41. Giese A, Bjerkvig R, Berens ME, et al. Cost of migration: invasion of malignant gliomas and implications for treatment. J Clin Oncol. 2003;21:1624-36.

42. Xie Q, Mittal S, Berens ME. Targeting adaptive glioblastoma: an overview of proliferation and invasion. Neuro Oncol. 2014;16:1575-84.

\section{Figures}


HIF-1 $\alpha$

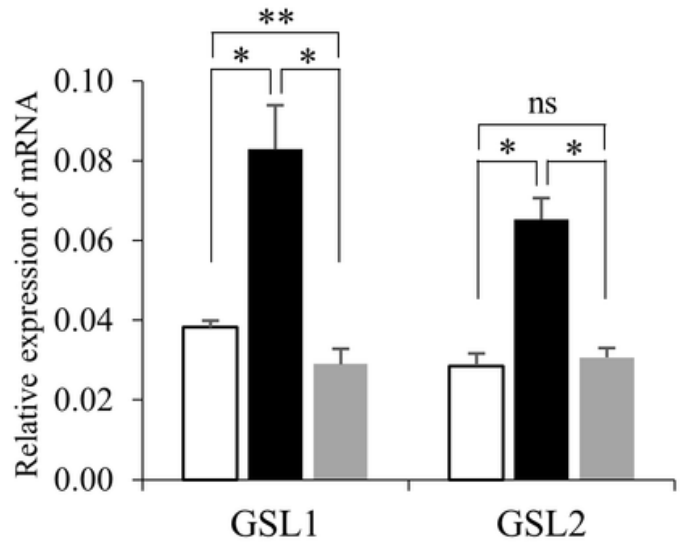

CD44

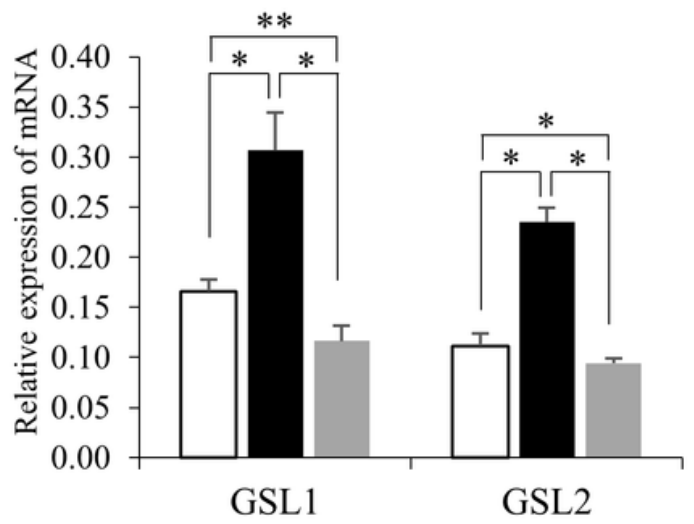

HIF- $2 \alpha$
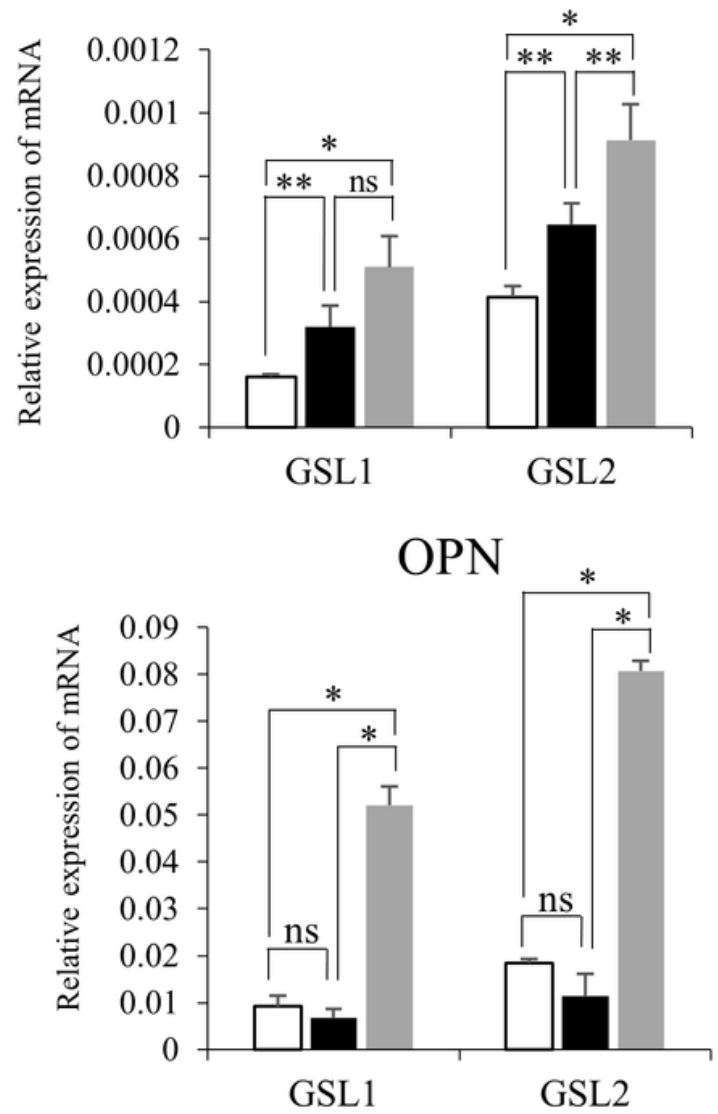

\section{Figure.1}

Normoxia

$1 \%$ Hypoxia

5\% Hypoxia

Figure 1

Effect of hypoxia on the expression of HIFs, CD44, and OPN in GSC lines. The mRNA expression of each molecule in both GSC lines was examined by qRT-PCR under three different oxygen concentrations, normoxia ( $21 \% 02)$, severe hypoxia, ( $1 \% 02)$, and moderate hypoxia $(5 \% 02)$. Values are relative expression of HIF-1a, HIF-2a, CD44, and OPN mRNA normalized to GAPDH mRNA. ${ }^{\star} \mathrm{P}<0.01{ }^{\star *} \mathrm{P}<0.05$. HIF, hypoxia-inducible factor; GSC, glioma stem like cell. 
A.

HIF-1 $\alpha$

CD44

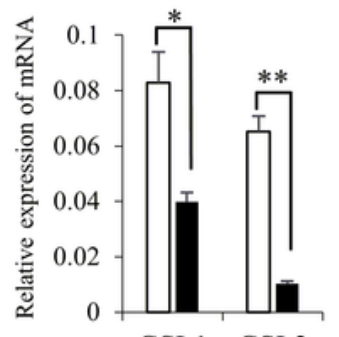

GSL1 GSL2

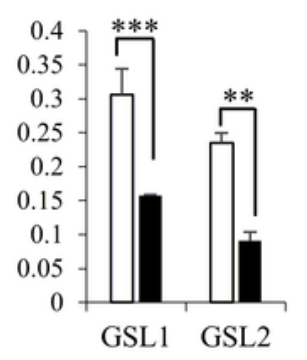

Control

A $1 \%$ Hypoxia)

B.

GSL1

GSL2

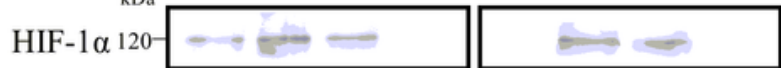

CD44

$\beta$-actin

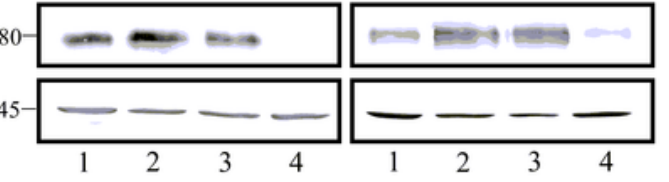

1: normoxia without siRNA

2: $1 \%$ Hypoxia without siRNA

3: $1 \%$ Hypoxia with control siRNA

4: $1 \%$ Hypoxia with HIF- $1 \alpha$ siRNA

C.
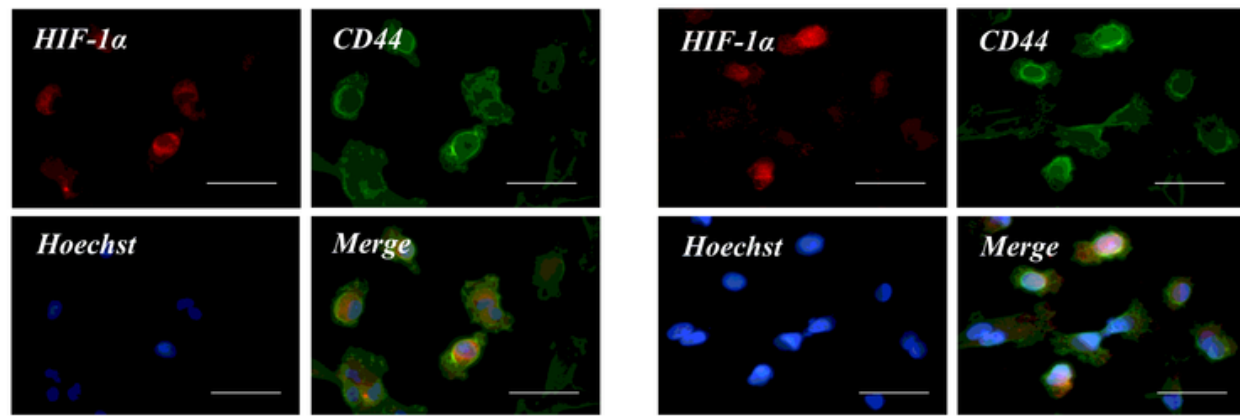

GSL1
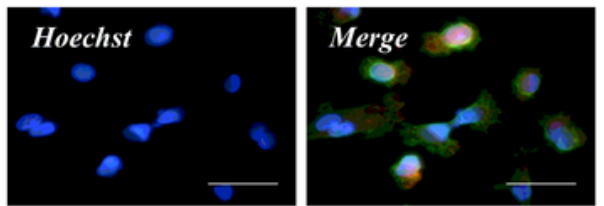

GSL2

D.

HIF- $2 \alpha$

OPN

E. GSL1 GSL2
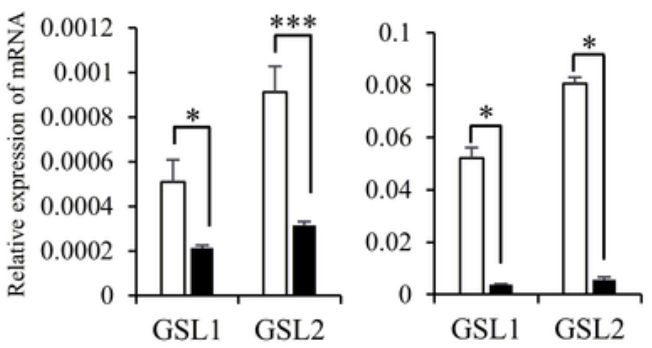

HIF-2 $\alpha 120-$
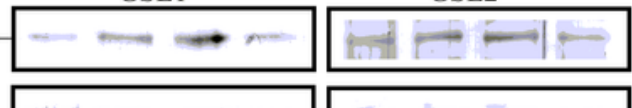

OPN

$\beta$-actin
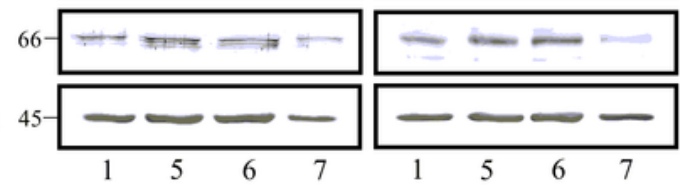

1: same above

5: $5 \%$ Hypoxia without siRNA

6: $5 \%$ Hypoxia with control siRNA

7: $5 \%$ Hypoxia with HIF- $2 \alpha$ siRNA

\section{F.}
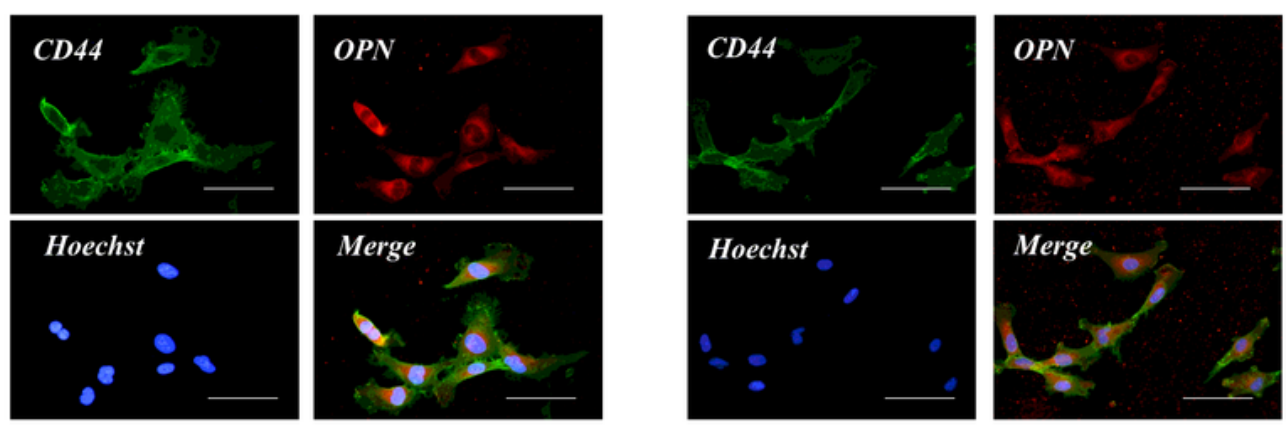

Figure.2

GSL1
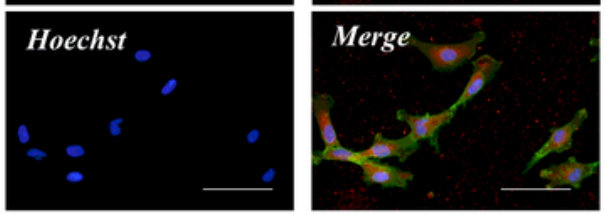

GSL2

\section{Figure 2}

Up-regulation of CD44 and OPN expression under different hypoxic conditions. (A) HIF-1 a siRNA significantly inhibited the expression of CD44 in both GSL1 and GSL2 cells under the condition of $1 \% 02$ hypoxia. ${ }^{*} P<0.05 * * P<0.001{ }^{* * *} P<0.005$. (B) Western blot analysis demonstrating the silencing effect of HIF-1a siRNA on the expression of HIF-1a and CD44 in two GSC lines. (C) Double-labeling immunofluorescence demonstrating co-localization of CD44 (green) and HIF-1a (red) under the condition 


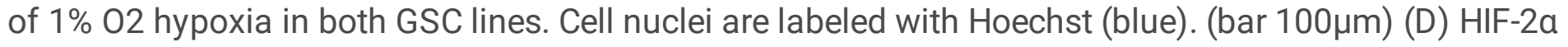
siRNA significantly inhibited the expression of OPN in both GSL1 and GSL2 cells under the condition of $5 \% 02$ hypoxia. ${ }^{*} P<0.05 * * P<0.001 * \star * P<0.005$. (E) Western blot analysis demonstrating silencing of HIF$2 a$ expression by siRNA and OPN expression in two GSC lines. (F) Double-labeling immunofluorescence demonstrating co-localization of CD44 (green) and OPN (red) under the condition of $5 \% 02$ hypoxia in

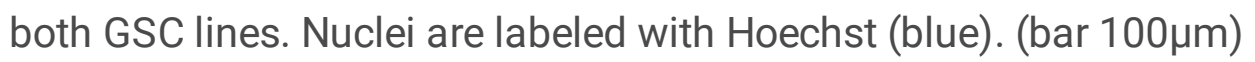

A. Tumor tissues

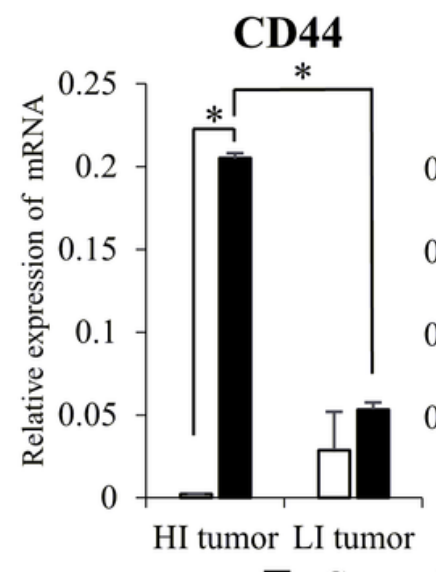

Core

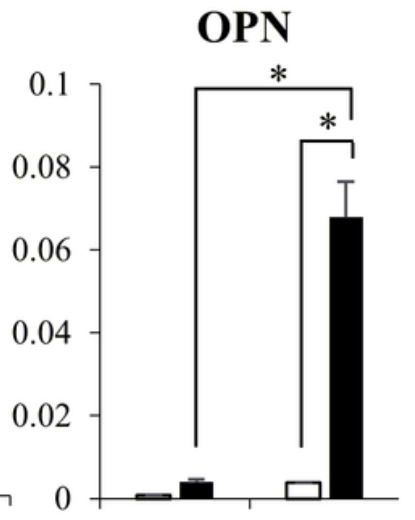

HI tumor LI tumor

Periphery
B. HI tumor (periphery)
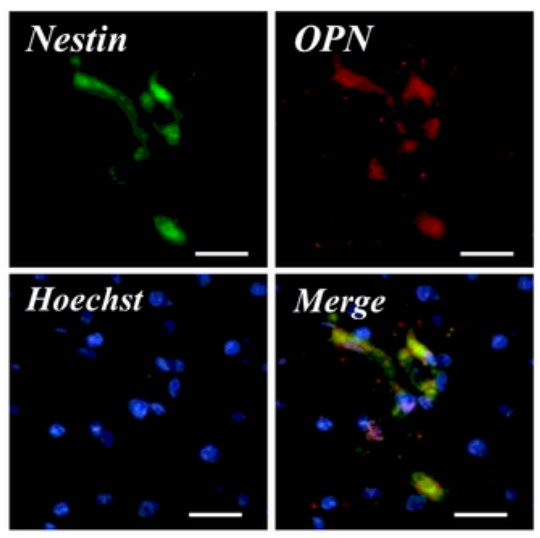

D.

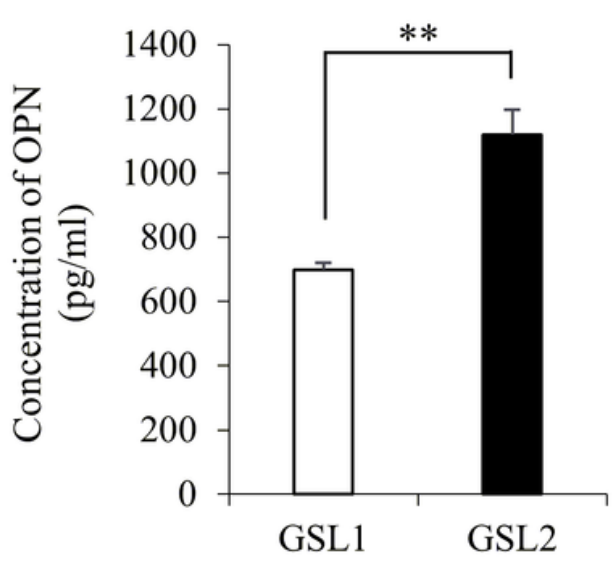

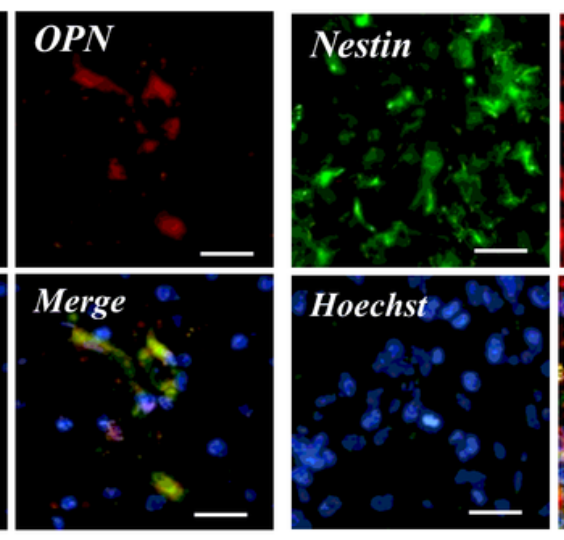

HI: high invasive

Extracellular fluid

Figure. 3

C.

Glioma stem-like cells

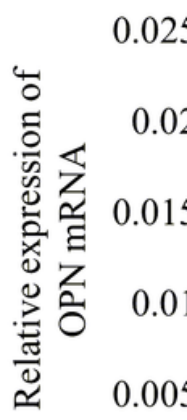

0

GSL1

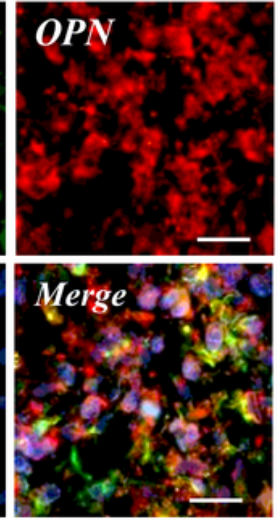

LI: low invasive

LI tumor (periphery)

\section{Figure 3}

Expression of OPN in GBM tissues and GSCs and secretion of OPN. (A) Both highly invasive GBM (HI tumor) and low-invasiveness GBM (LI tumor) expressed more OPN in the periphery of the tumor than in the core. The peripheral tissues of LI tumors expressed OPN at a significantly higher level than in the core of either $\mathrm{LI}$ or HI tumors. ${ }^{*}<<0.001$. (B) Double-labeling immunofluorescence demonstrating colocalization of OPN (red) and Nestin (green) in the periphery of $\mathrm{HI}$ and $\mathrm{LI}$ tumors. Nuclei are labeled with

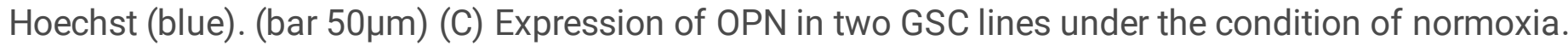
Values are relative expression of OPN mRNA normalized to that of GAPDH mRNA. ${ }^{* * P<0.01 . ~}$ Extracellular concentration of OPN in conditioned medium of two GSC lines. ${ }^{*} \mathrm{P}<0.01$. 
A.

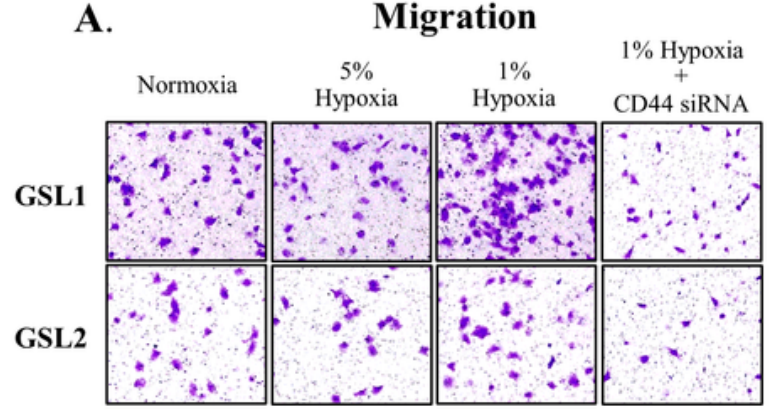

Migration

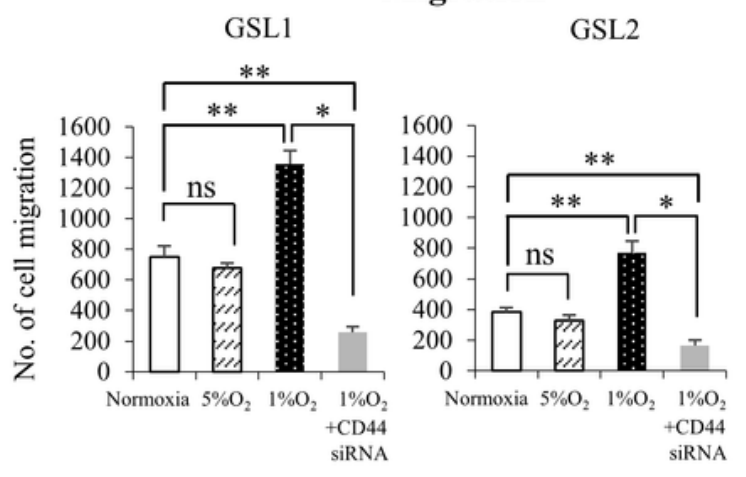

B.

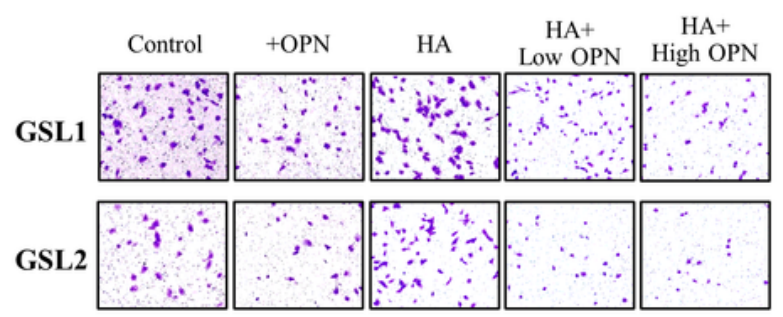

Migration

GSL1

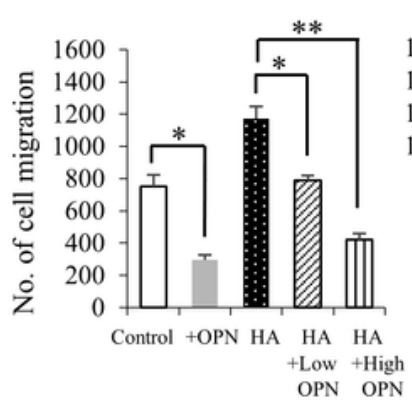

Figure. 4

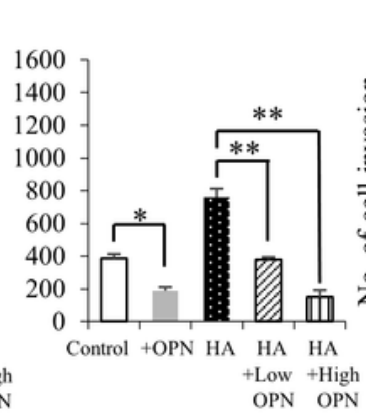

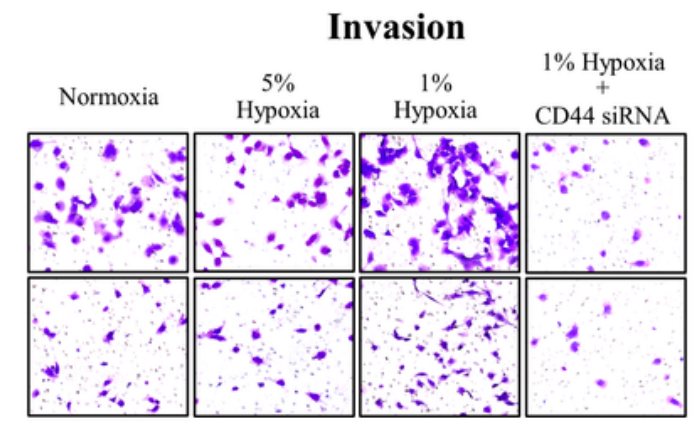

Invasion

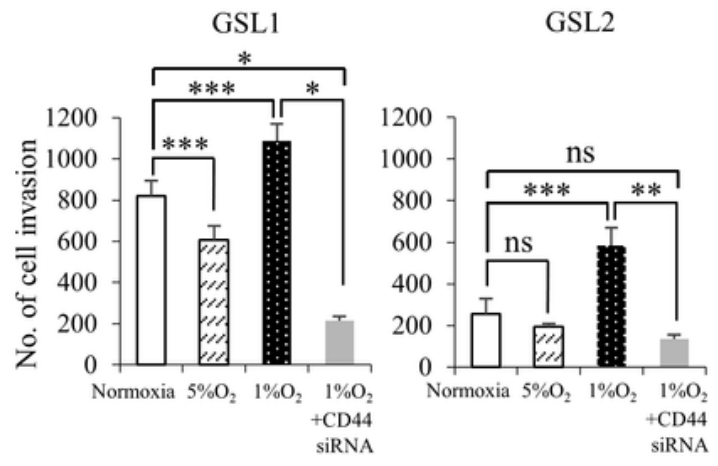

\section{Invasion}

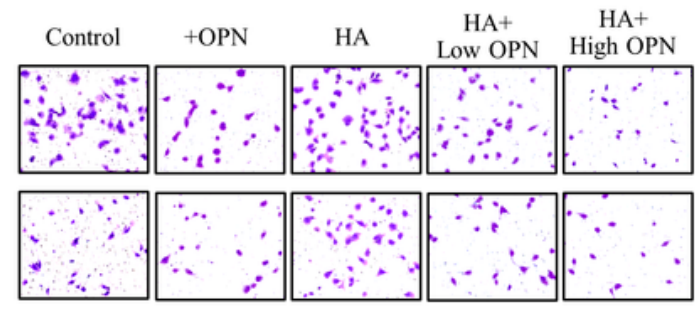

Invasion

GSL1

GSL2
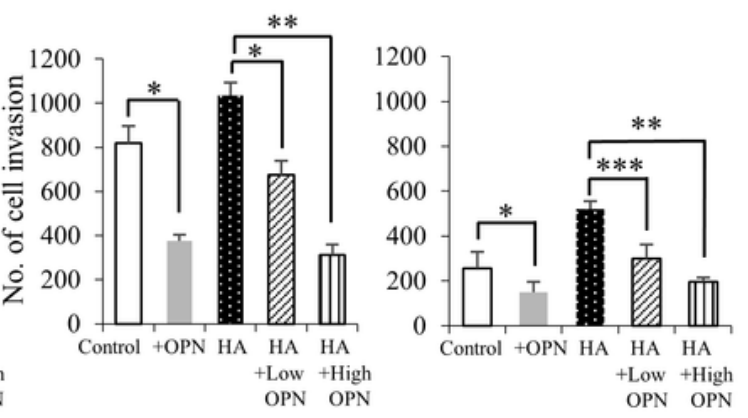

\section{Figure 4}

Enhancing effect of hypoxia and reducing effect of OPN on GSC migration and invasion. (A) Effects of hypoxia and CD44 on in vitro cell migration and invasion of two GSC lines. Under the condition of $1 \% 02$ hypoxia, the migration and invasion of both GSC lines was markedly increased, whereas $5 \% 02$ hypoxia had no effect on either migration or invasion and rather decreased the invasiveness of GSL1 cells. CD44 silencing via siRNA significantly inhibited the 1\% 02 hypoxia-induced increase in GSC migration and 
invasion. Upper panel shows tumor cells that had migrated or invaded to the lower side of the filter membrane (stained with crystal violet). ${ }^{*} P<0.001{ }^{\star}{ }^{*} \mathrm{P}<0.005 * \star * P<0.05$. (B) Effect of OPN on HA-induced migration and invasion of two GSC lines. OPN inhibited CD44-mediated migration and invasion in both GSC lines under the normoxic condition. Addition of $\mathrm{HA}(0.5 \mathrm{mg} / \mathrm{ml})$ enhanced the migration and invasion of both GSC lines, whereas OPN administration (low:0.2 $\mu \mathrm{g} / \mathrm{ml}$, high: $5.0 \mu \mathrm{g} / \mathrm{ml}$ ) suppressed the enhancement of migration and invasion in a dose-dependent manner. ${ }^{*} P<0.005 * * P<0.001{ }^{*} * * P<0.05$.
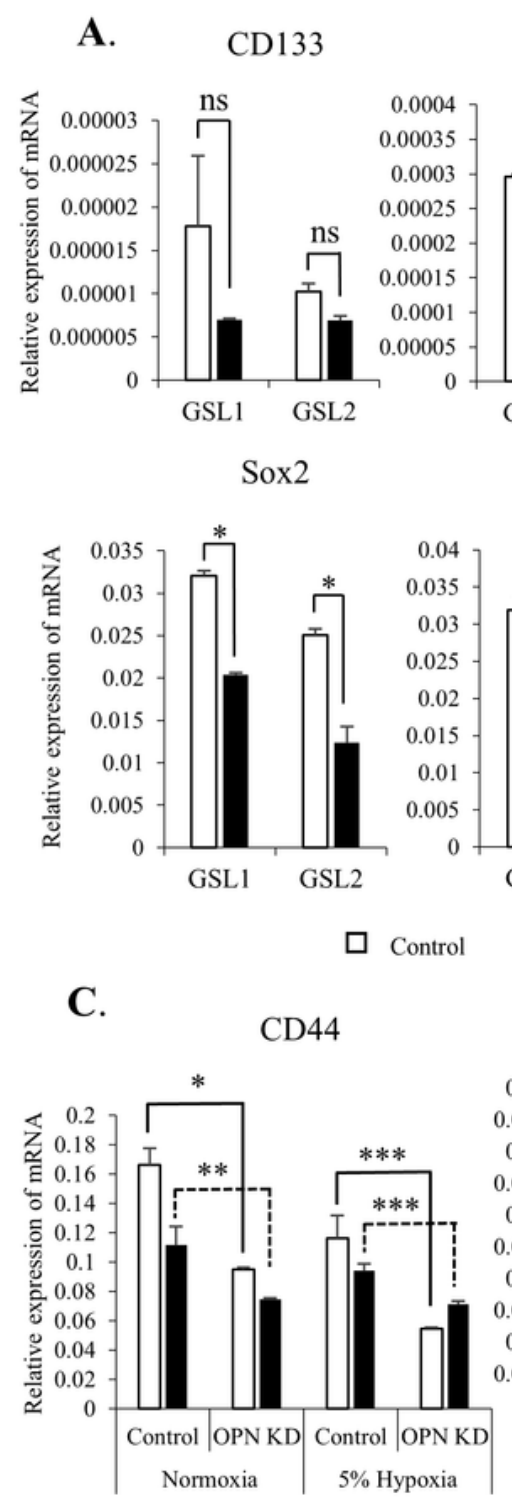

D.

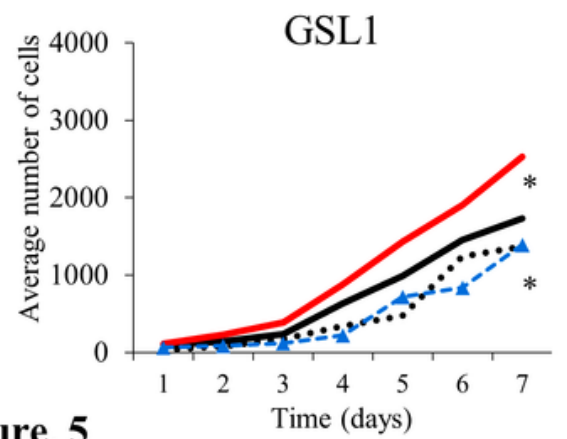

Figure. 5

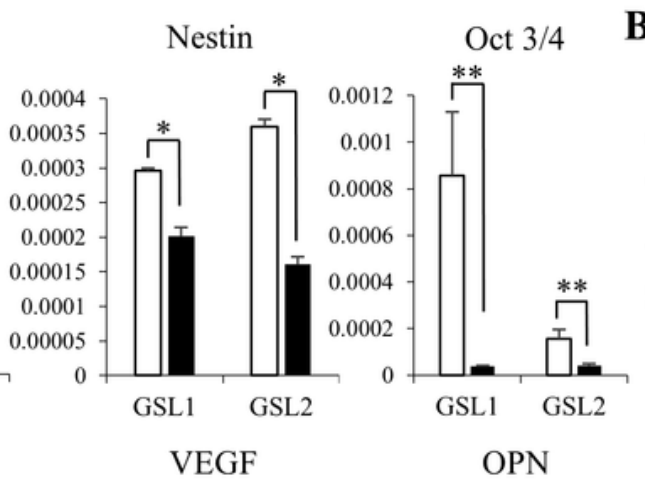

B. GSL1
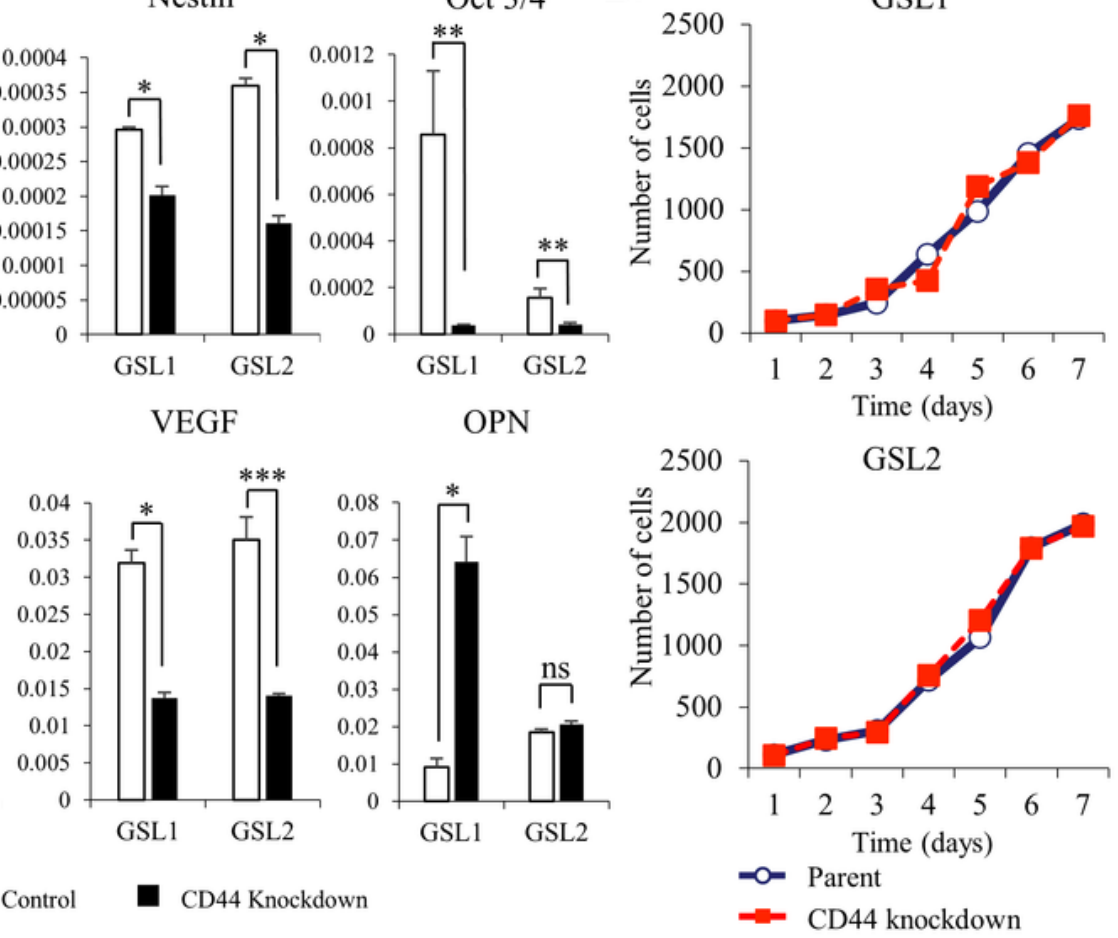
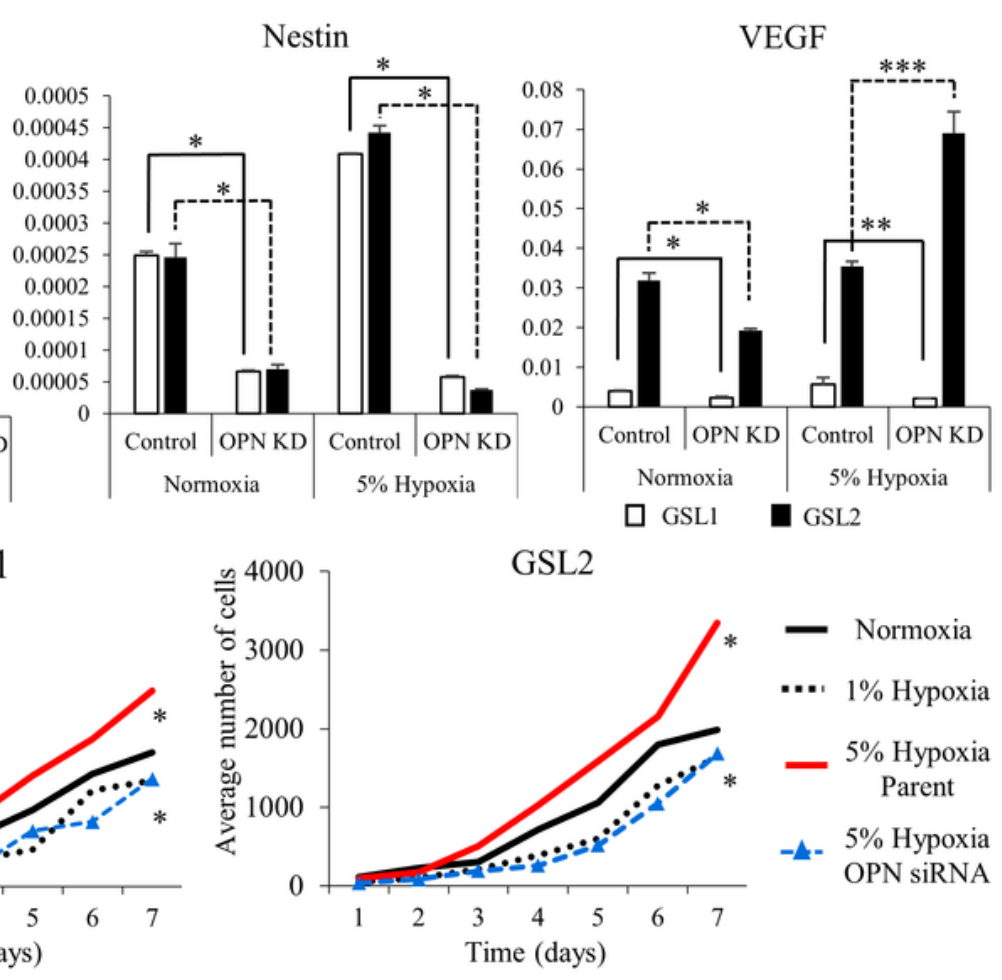

Figure 5 
Effect of CD44 or OPN knockdown on the expression of other markers and cell proliferation. (A) Effect of CD44 knockdown on the expression of neural stem cell markers and VEGF. Knockdown of CD44 using shRNA significantly decreased the expression of all stem cell markers except CD133 examined in both GSC lines. ${ }^{*} P<0.001,{ }^{*} P<<0.05$, ${ }^{\star * *} P<0.005$. (B) Effect of CD44 knockdown on GSC proliferation. Graphs show growth curves of GSCs with and without CD44 knockdown. CD44 knockdown had no effect on the proliferation of the GSC lines in cell culture. (C) Effect of OPN inhibition on the expression of CD44, Nestin, and VEGF under normoxia and 5\% 02 hypoxia. Silencing of OPN expression using siRNA significantly decreased the expression of CD44 and Nestin in both GSC lines and increased the expression of VEGF only in GSL2 cells. ${ }^{*} P<0.001$, ${ }^{*} P<0.05 * \star * P<0.005$. (D) Effects of hypoxia and OPN silencing on GSC proliferation. Under the condition of 5\% 02 hypoxia, the growth rate of both GSC lines increased significantly compared with GSCs cultured under the normoxic condition (control). Inhibition of OPN expression significantly decreased the growth rate of both GSC lines compared with the control. $* P<0.001$.

A.
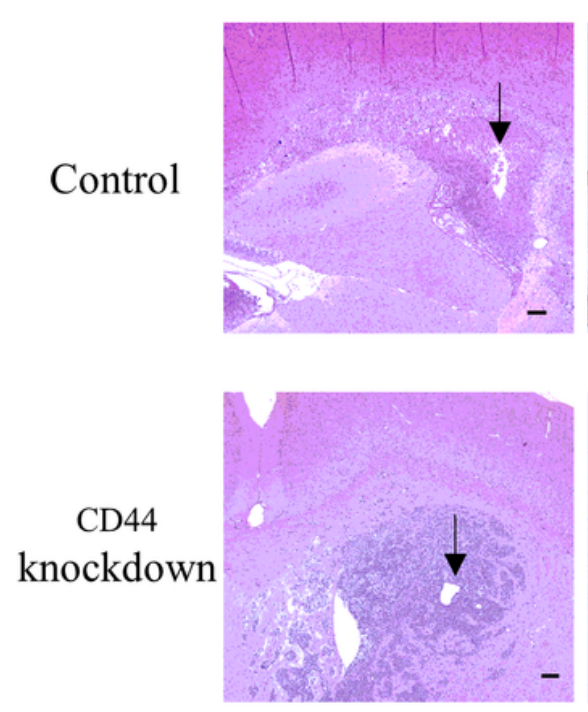

C.
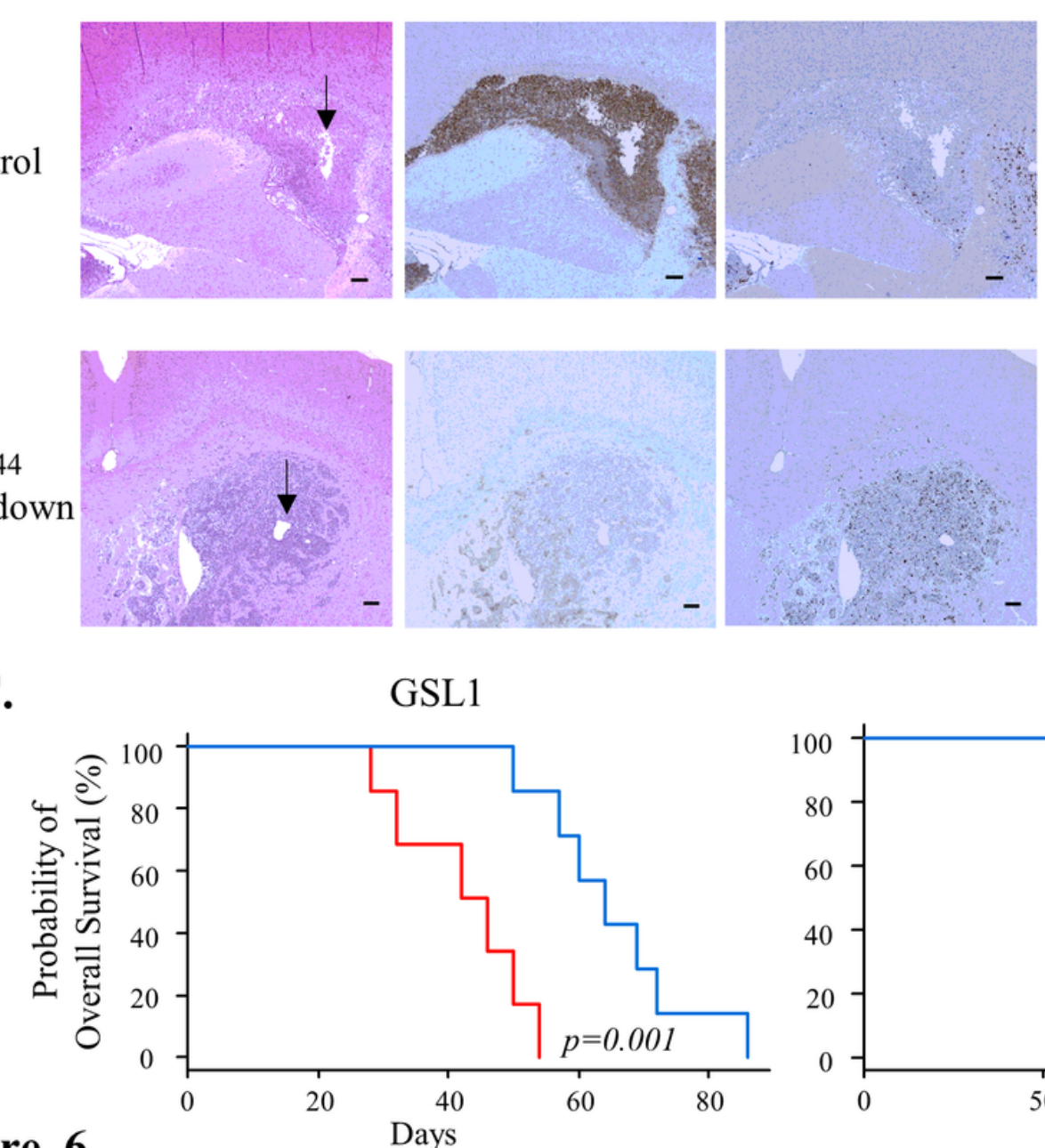

GSL1

Figure. 6

Days
Ki67

B.

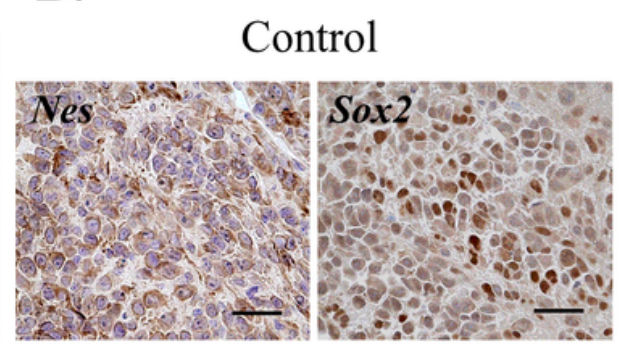

\section{CD44 knockdown}

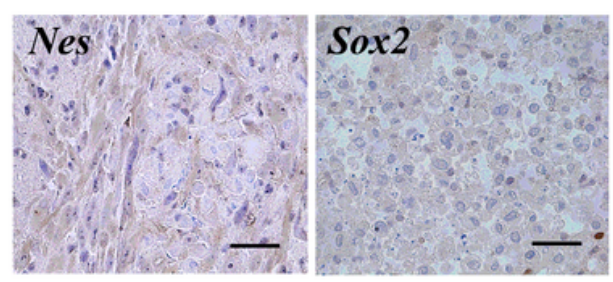

GSL2

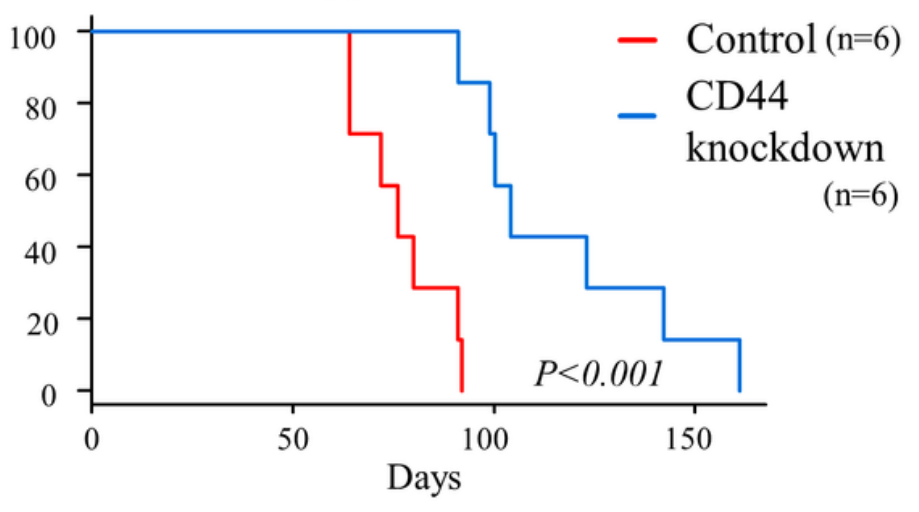

\section{Figure 6}


Histologic features and survival curves of CD44-knockdown GSCs transplanted mice. (A) Microscopic images of tumors generated in mouse brain via transplantation of GSCs with or without CD44 knockdown (×40) (upper panel, control; lower panel, CD44 knockdown). Histologic examination of control specimens at low magnification indicated an unclear tumor margin with diffuse infiltration of tumor cells around the tumor, particularly marked invasion into the corpus callosum (H\&E) (left column). In contrast, transplantation of GSCs with CD44 knockdown generated a well-demarcated tumor around the GSC injection site without extensive infiltration of tumor cells into the surrounding brain tissues (left column). Arrow: needle track of tumor injection. Almost all tumor cells in the control were positively stained with anti-CD44 antibody, but almost no tumor cells stained positive for CD44 in the tumor with CD44

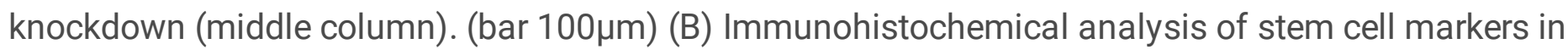
tumors generated by GSC transplantation $(\times 400)$. Both Nestin and Sox 2 were positively immunostained in the tumor generated by transplantation of control GSCs (upper panel), but neither marker stained

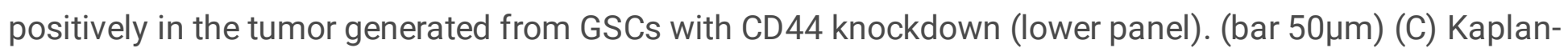
Meier overall survival curves of mice that received transplantation of GSL1 or GSL2 cells. Mice received transplants of GSCs with CD44 knockdown exhibited significantly longer survival than the control for both GSL1 $(P=0.001)$ and GSL2 $(P<0.001)$.

\section{High-invasiveness type}

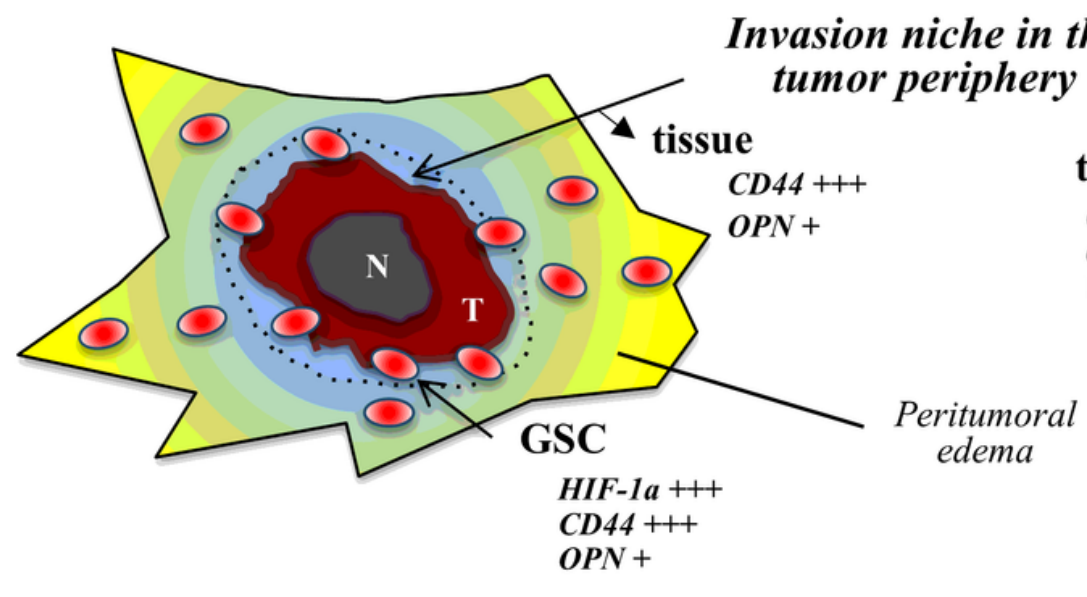

Migration/Invasion

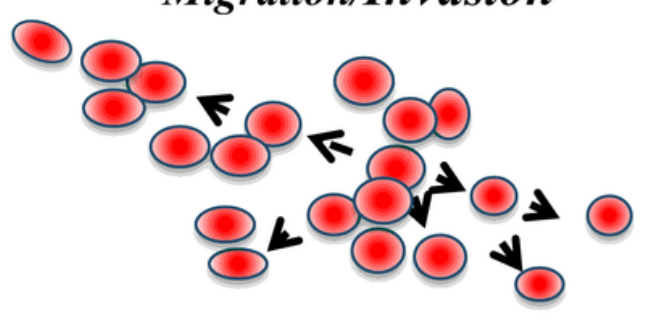

(HIF-1 $/$ /CD44T)

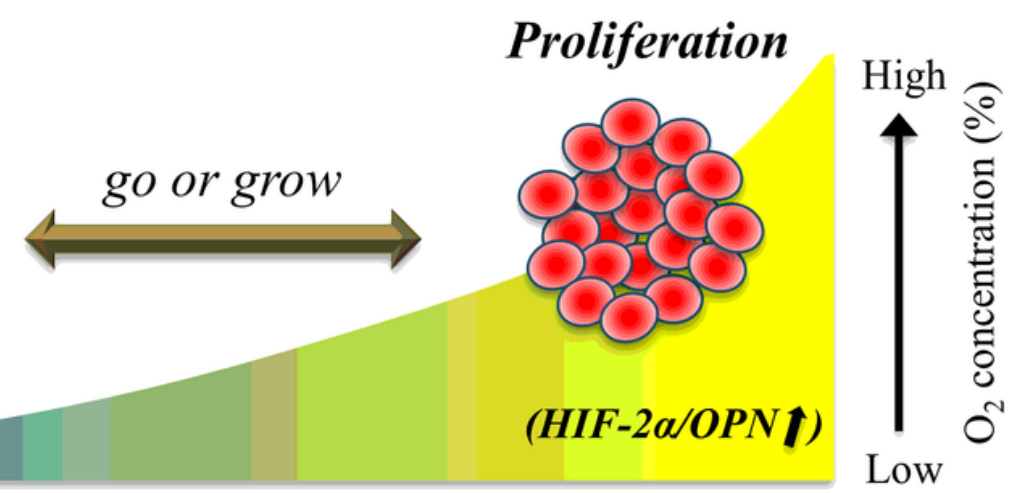

\section{Low-invasive/highly- proliferative type}

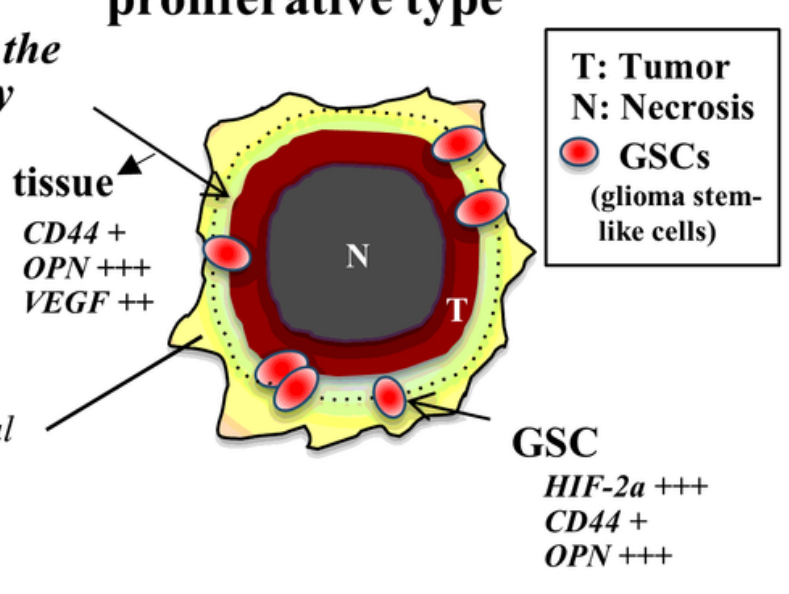




\section{Figure 7}

Hypothetical illustration of two GBM phenotypes, including activation of CD44 and OPN by hypoxia. Under severe hypoxia ( 1\% 02), CD44 expression is up-regulated via the activation of HIF-1a, which strongly promotes GSC migration and invasion, generating the highly invasive GBM phenotype. Under moderate hypoxia (3 5\% 02), OPN expression is up-regulated via HIF-2a, which inhibits CD44-mediated migration and invasion and enhances GSC proliferation, resulting in the low-invasiveness/highly proliferative GBM phenotype. Switching of phenotypes can be induced by changing the oxygen concentration in the tumor microenvironment.

\section{Supplementary Files}

This is a list of supplementary files associated with this preprint. Click to download.

- ARRIVEchecklist.docx

- FigureS1.tif

- Figures2.TIF

- Figures3.tif

- Figures4.tif

- additionalfile.pdf 